\title{
Epicutaneous Staphylococcus aureus induces IL-36 to enhance IgE production and ensuing allergic disease
}

\author{
Garrett J. Patrick, ${ }^{1}$ Haiyun Liu, ${ }^{1}$ Martin P. Alphonse, ${ }^{1}$ Dustin A. Dikeman, ${ }^{1}$ Christine Youn, ${ }^{1}$ Jack C. Otterson, ${ }^{1}$ Yu Wang, ${ }^{1}$ \\ Advaitaa Ravipati, ${ }^{1}$ Momina Mazhar, ${ }^{1}$ George Denny, ${ }^{1}$ Roger V. Ortines, ${ }^{1}$ Emily Zhang, ${ }^{1}$ Robert J. Miller, ${ }^{1}$ Carly A. Dillen, ${ }^{1}$ \\ Qi Liu, ${ }^{1}$ Sabrina J. Nolan, ${ }^{1}$ Kristine Nguyen, ${ }^{1}$ LeeAnn Marcello, ${ }^{1}$ Danh C. Do, ${ }^{2}$ Eric M. Wier, ${ }^{1}$ Yan Zhang, ${ }^{2}$ Gary Caviness, ${ }^{3}$ \\ Alexander C. Klimowicz, ${ }^{3}$ Diane V. Mierz, ${ }^{3}$ Jay S. Fine,${ }^{3}$ Guangping Sun, ${ }^{4}$ Raphaela Goldbach-Mansky, ${ }^{5}$ Alina I. Marusina, ${ }^{6}$ \\ Alexander A. Merleev, ${ }^{6}$ Emanual Maverakis, ${ }^{6}$ Luis A. Garza, ${ }^{1}$ Joshua D. Milner, ${ }^{7}$ Peisong Gao, ${ }^{2}$ Meera Ramanujam, ${ }^{3}$ \\ Ernest L. Raymond, ${ }^{3}$ Nathan K. Archer, ${ }^{1}$ and Lloyd S. Miller ${ }^{1}$
}

'Department of Dermatology and 'Division of Allergy and Clinical Immunology, Johns Hopkins University School of Medicine, Baltimore, Maryland, USA. ${ }^{3}$ Immunology \& Respiratory Diseases Research, Boehringer Ingelheim Pharmaceuticals Inc., Ridgefield, Connecticut, USA. ${ }^{4}$ Laboratory of Allergic Diseases, NIAID, NIH, Bethesda, Maryland, USA. ${ }^{5}$ Translational Autoinflammatory Disease Section (TADS) NIAID, NIH, Bethesda, Maryland, USA. ${ }^{6}$ Department of Dermatology, School of Medicine, University of California, Davis, Sacramento, California, USA. ${ }^{7}$ Department of Pediatrics, Columbia University Irving Medical Center, New York, New York, USA.

IgE induced by type 2 immune responses in atopic dermatitis is implicated in the progression of atopic dermatitis to other allergic diseases, including food allergies, allergic rhinitis, and asthma. However, the keratinocyte-derived signals that promote IgE and ensuing allergic diseases remain unclear. Herein, in a mouse model of atopic dermatitis-like skin inflammation induced by epicutaneous Staphylococcus aureus exposure, keratinocyte release of IL-36 $\alpha$ along with IL-4 triggered B cell IgE class-switching, plasma cell differentiation, and increased serum IgE levels-all of which were abrogated in IL-36R-deficient mice or anti-IL-36R-blocking antibody-treated mice. Moreover, skin allergen sensitization during S. aureus epicutaneous exposure-induced IL-36 responses was required for the development of allergen-specific lung inflammation. In translating these findings, elevated IL-36 cytokines in human atopic dermatitis skin and in IL-36 receptor antagonistdeficiency patients coincided with increased serum IgE levels. Collectively, keratinocyte-initiated IL-36 responses represent a key mechanism and potential therapeutic target against allergic diseases.

\section{Introduction}

Allergic diseases have been increasing in recent decades and currently affect nearly $20 \%$ of the human population. These include 200 million people with atopic dermatitis (AD) (including up to $20 \%$ of children and $5 \%$ of adults in the United States), 250 million people with food allergies, 400 million people with allergic rhinitis, and 200-250 million people with asthma (1). Patients with allergic diseases often suffer from more than one of these conditions, indicating an association and suggesting that common mechanisms contribute to allergic disease pathogenesis (2-4). In general, the pathogenesis of allergic diseases involves type 2

Authorship note: GJP, HL, NKA, and LSM contributed equally to this work. Conflict of interest: GC, ACK, DVM, JSF, MR, and ELR are full-time employees of Boehringer Ingelheim, which is developing therapeutics targeting IL-36R inhibition in patients with atopic dermatitis and other inflammatory conditions. LSM is a full-time employee of Janssen Pharmaceuticals and holds Johnson \& Johnson stock. LSM received prior grant support from AstraZeneca, Pfizer, Boehringer Ingelheim, Regeneron Pharmaceuticals, and Moderna Therapeutics, was a paid consultant for Armirall and Janssen Research and Development, was on the scientific advisory board of Integrated Biotherapeutics, and is a shareholder of Noveome Biotherapeutics, which are all developing therapeutics against infections (including S. aureus and other pathogens) and/or inflammatory conditions.

Copyright: ( 2021, American Society for Clinical Investigation.

Submitted: August 17, 2020; Accepted: December 16, 2020; Published: March 1, 2021.

Reference information: J Clin Invest. 2021;131(5):e143334.

https://doi.org/10.1172/JCl143334. immune responses and allergen-specific $\operatorname{IgE}$ that activate mast cells and basophils to promote aberrant inflammation (5). Certain studies have supported the concept of the atopic march, in which initial environmental allergen sensitization in the barrier-defective skin of patients with $\mathrm{AD}$ is thought to trigger allergen-specific IgE production, which promotes the subsequent development of other allergic diseases (2-4). Although this concept has been challenged (as allergic diseases do not always occur sequentially) (3, 6), a greater understanding of the overarching pathogenic mechanisms is essential to provide more specific therapeutic targets.

The skin barrier defects in patients with $\mathrm{AD}$ have been attributed to loss-of-function mutations and/or decreased expression of filaggrin as well as other components that maintain the epidermal barrier (e.g., loricrin and involucrin) $(7,8)$. The affected skin of patients with AD also has markedly increased Staphylococcus aureus colonization that further exacerbates skin inflammation through the activity of $S$. aureus-derived cytolytic toxins, superantigens, and proteases (9-11). In this setting of skin barrier defects and dysbiosis in $\mathrm{AD}$, there is also markedly increased production of IL-33, thymic stromal lymphopoietin (TSLP), and IL-25 that skew the immune response toward type 2 immunity with increased IgE production and allergic inflammation by activating dendritic cells, Th2 cells, group 2 innate lymphoid cells (ILC2s), mast cells, eosinophils, and basophils (2-4). Importantly, the keratinocytes in the inflamed epidermis in $\mathrm{AD}$ are thought to play a key role in orches- 
A

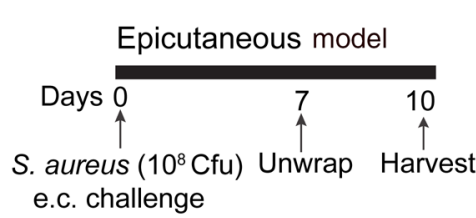

e.c. challenge
B

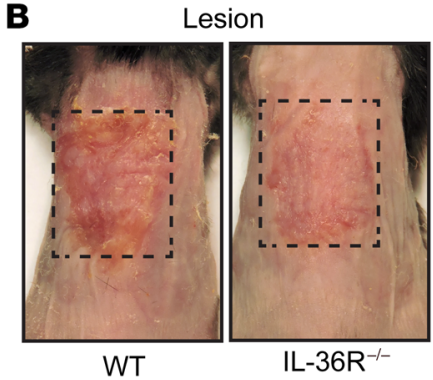

Disease score

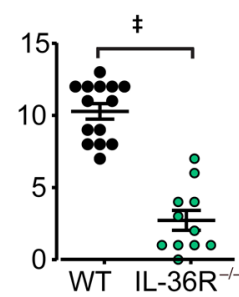

C

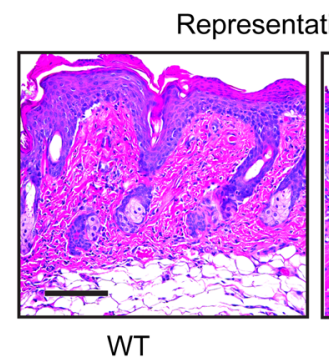

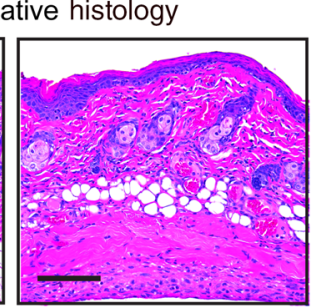

$\mathrm{IL}-36 \mathrm{R}^{-/-}$
Epidermis $(\mu \mathrm{m})$

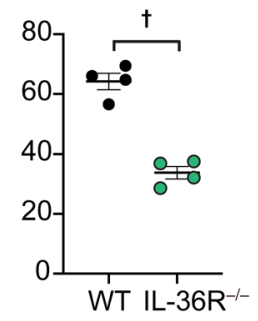

Figure 1. Markedly elevated serum IgE levels in response to $S$. aureus e.c. challenge is dependent on IL-36R signaling. The S. aureus e.c. model was performed in WT and IL-36R $\mathrm{R}^{-/-}$mice. (A) Timeline of S. aureus e.c. model. (B) Representative skin photographs and mean disease score \pm SEM ( $n=11-14$ / group) (C) Representative histology (H\&E stain) and mean epidermal thickness $(\mu \mathrm{m}) \pm \operatorname{SEM}(n=4 /$ group). Scale bars: $100 \mu \mathrm{m}$. (D) Mean serum lgE (ng/ $\mathrm{mL}) \pm \mathrm{SEM}$ ( $n=8-10 /$ group). (E) Mean serum IgG1 and IgG2a levels $(\mathrm{ng} / \mathrm{mL}) \pm$ SEM ( $n=4$ /group). ${ }^{\dagger} P<0.01,{ }^{\ddagger} P<0.001$ between indicated groups, as calculated by 2-tailed Student's $t$ test (B and C) or 2-way ANOVA (D and E). Results are representative of 2 independent experiments.

\section{Serum $\lg E(\mathrm{ng} / \mathrm{mL})$}

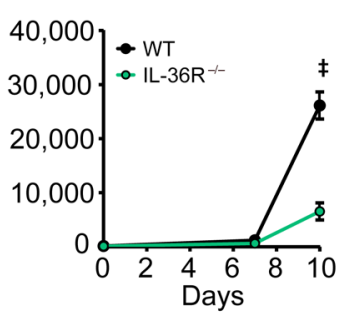

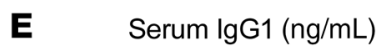

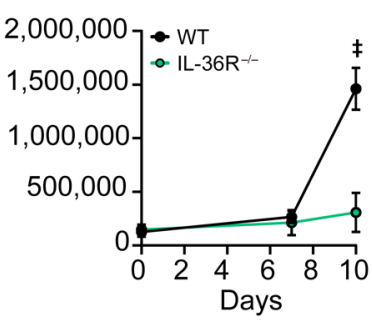

trating these pathogenic immune responses because they are the major cellular source of IL-33 $(12,13)$ and TSLP (14-16).

Although the type 2 immune responses in patients with AD likely contribute to the association and increase the susceptibility to other IgE-mediated allergic diseases (especially allergic asthma and food allergies), a keratinocyte-derived signal that directly triggers B cells to induce IgE production has yet to be identified. Indeed, TSLP and IL-33, which are produced by keratinocytes, promote early B cell development rather than IgE production $(17,18)$. Therefore, in this study, we sought to determine whether keratinocytes in the setting of $\mathrm{AD}$-inflamed skin could provide a mechanism to directly trigger B cells to induce IgE class switching, increased production of $\mathrm{IgE}$, and progression to IgE-mediated allergic lung inflammation.

\section{Results}

S. aureus epicutaneous exposure induces IL-36-dependent increased serum IgE in mice. To investigate for a role of keratinocytes in directly promoting B and plasma cell (PC) production of IgE, a mouse model of $S$. aureus epicutaneous (e.c.) exposure that produces AD-like skin inflammation (19-21) was employed in which a $S$. aureus (methicillin-resistant $S$. aureus [MRSA] strain LAC4303) soaked gauze pad was applied to the backs of mice and secured with adhesive bandages (Figure 1A). The bandages were removed on day 7 and WT mice developed skin inflammation (disease score) and increased epidermal thickness, which were almost entirely attenuated in IL-36 $\mathrm{R}^{-/-}$mice (Figure $1, \mathrm{~B}$ and C) (despite

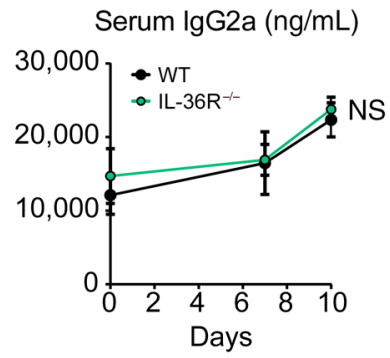

similar bacterial burden [Supplemental Figure 1A; supplemental material available online with this article; https://doi.org/10.1172/ JCI143334DS1], consistent with prior reports, refs. 19, 20). However, on day 10, WT mice developed substantially increased serum IgE levels $(>20,000 \mathrm{ng} / \mathrm{mL}$ ) and serum IgG1 levels whereas serum IgG2a levels were similar between WT and IL-36R $\mathrm{R}^{-/}$mice (Figure 1, D and E). This finding indicates that IL-36R signaling promotes class-switching of Th2-induced IgG1/IgE production but not Th1-induced IgG2a production (22).

IL-36R-induced serum IgE in mice is dependent on the anatomical localization of $S$. aureus exposure in the skin. To determine whether the increased serum IgE levels observed following $S$. aureus e.c. exposure required surface contact between $S$. aureus and keratinocytes, an alternative $S$. aureus intradermal (i.d.) challenge model was performed (Figure 2A), as previously described $(23,24)$ using a bioluminescent $S$. aureus strain (USA300 LAC::lux, derived from the same parental MRSA strain as in Figure 1, A-E). In contrast to $S$. aureus e.c. exposure (Figure 1, A-E), in the $S$. aureus i.d. challenge model there were no differences in skin lesion sizes, bacterial burden (measured by in vivo bioluminescence imaging) (Figure $2 \mathrm{~B}$ ), and levels of serum IgE (which were almost completely absent), IgG1 and IgG2a between WT and IL-36R $\mathrm{R}^{-/-}$mice (Figure 2, C and D). Therefore, only the S. aureus e.c. model induced the substantially increased serum IgE levels. To further determine the specificity of the IgE response to IL-36R signaling, the $S$. aureus e.c. model was performed in IL-1 $1 \beta^{-/-}$mice. In these experiments, $\mathrm{IL}-1 \beta^{-/-}$mice were used as a comparison for $\mathrm{IL}-36 \mathrm{R}^{-/-}$mice because 
A

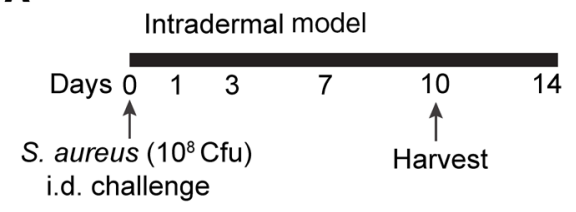

B Total lesion size $\left(\mathrm{cm}^{2}\right)$

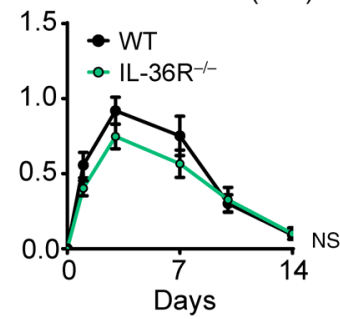

In vivo $\mathrm{BLI}(\log$ scale)

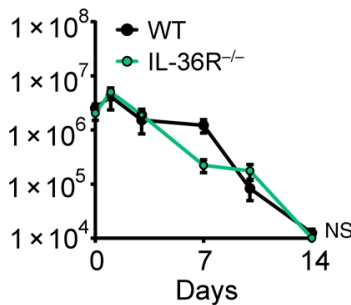

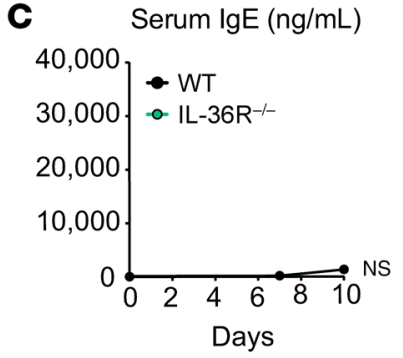
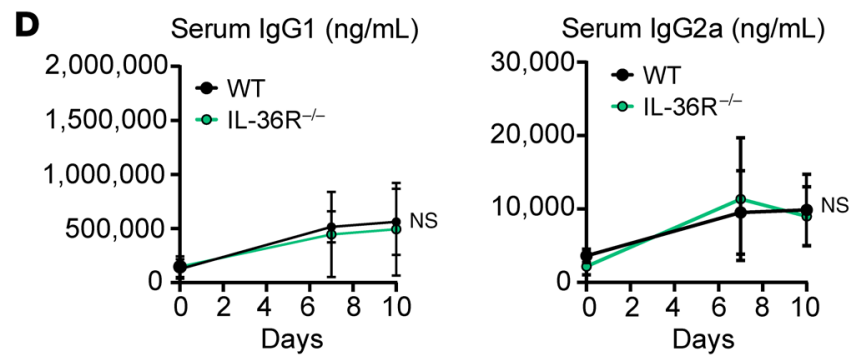

E

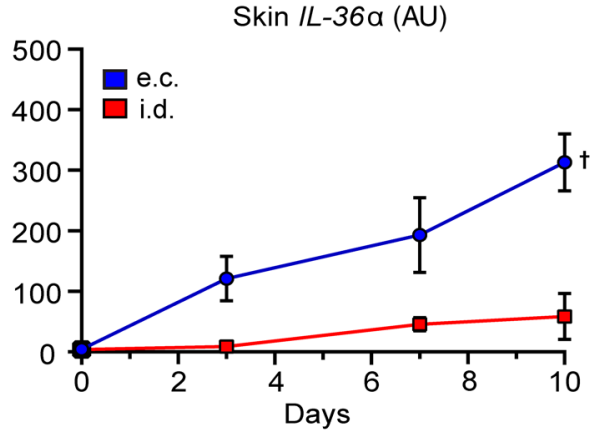

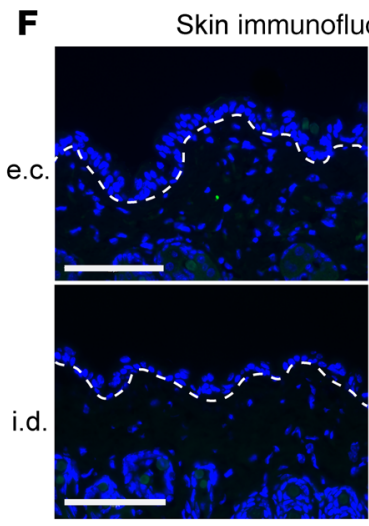

Day 0

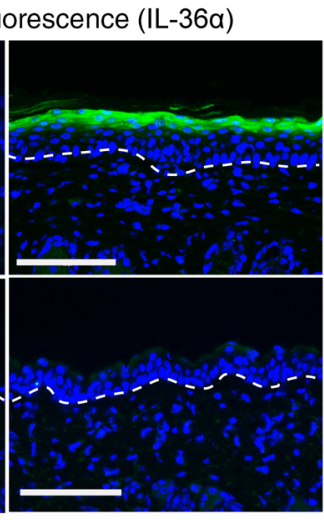

Day 10

Figure 2. IL-36 responses are dependent on the anatomical localization of S. aureus challenge. The S. aureus i.d. model (A-F) or e.c. model (E and F) was performed in WT and IL-36R cence imaging signals (total flux; photons $/ \mathrm{s}) \pm$ SEM ( $n=5 / g r o u p)$. (C) Mean serum lgE ( $\mathrm{ng} / \mathrm{mL})(n=4-5 / g r o u p)$ and (D) lgG1 and lgG2a levels (ng/mL) \pm SEM ( $n=4$ /group). (E) Mean mRNA levels (AU) \pm SEM of transcripts for IL-36 $\alpha \pm$ SEM ( $n=4-9 /$ group). (F) Representative immunofluorescence microscopy sections on days 0 and 10 labeled with anti-IL-36 (green) and DAPI (blue). Scale bars: $100 \mu \mathrm{m}$; dashed line indicates dermoepidermal junction. ${ }^{\dagger} P<0.01$ between indicated groups, as calculated by 2-way ANOVA. Results are representative of 2 independent experiments.

IL-1 $\beta$ is an alternative IL-1 cytokine family member that has previously been implicated in the host defense against $S$. aureus skin infections (23). In contrast to the results with the $S$. aureus e.c. model in IL-36 $\mathrm{R}^{-/-}$mice (Figure $1, \mathrm{~A}-\mathrm{E}$ ), in IL-1 $\beta^{-/-}$mice there were no differences in skin disease score, epidermal thickness, or serum IgE levels compared with WT mice (Supplemental Figure 1, B-D). In addition, although IL-36 $\alpha$, IL-36 $\beta$, and IL-36 $\gamma$ cytokines are all known to trigger activation of IL-36R signaling (25), only $I L-36 \alpha$ transcript levels in the skin were increased in the $S$. aureus e.c. model (20). Notably, increased $I L-36 \alpha$ transcript levels and IL-36 $\alpha$ protein expression in keratinocytes were only observed in the $S$. aureus e.c. model but not the $S$. aureus i.d. challenge model (Figure 2, E and F and Supplemental Figure 2), providing further evidence that the IL-36R-mediated IgE responses were likely dependent on the anatomical location of the $S$. aureus exposure.

Since $S$. aureus induces IL-33 and TSLP release from keratinocytes $(26,27)$, we also evaluated whether IL33 and TSLP transcripts were induced in primary mouse epidermal keratinocytes in vitro after 4 hours of culture with or without recombinant mouse IL-36 $\alpha$ (rIL-36 ) (Supplemental Figure 3, A and B). We found that transcripts for IL33 and TSLP were significantly increased in mouse keratinocytes in response to rIL-36 $\alpha$ compared with no treatment (control, $P<0.05$ ). In addition, we evaluated whether IL33 and $T S L P$ transcripts were induced in the $S$. aureus e.c. model on day 0 in naive mice (prior to $S$. aureus exposure) and on day 10 in WT and IL-36 $\mathrm{R}^{-/-}$mice (Supplemental Figure 3, C and D). There was virtually no induction of IL33 transcripts at baseline in naive WT mice (or naive $\mathrm{IL}^{-36 \mathrm{R}^{-/-}}$mice, data not shown). However, on day 10 of the $S$. aureus e.c. model, there was substantial induction of IL33 transcripts that was markedly attenuated ( $>50 \%$ reduction) in IL-36 $\mathrm{R}^{-1-}$ mice $(P<0.05$, Supplemental Figure $3 \mathrm{C})$. Similarly, there was virtually no induction of TSLP at baseline in naive WT mice (or naive IL-36R $\mathrm{R}^{-/}$mice, data not shown) (Supplemental Figure 3D). However, on day 10 of the S. aureus e.c. model, there was substantial induction of TSLP that was markedly attenuated (>60\% reduction) in $\mathrm{IL}-36 \mathrm{R}^{-/-}$mice $(P<0.05)$. These results suggested 
A Disease score Serum $\lg E(n g / m L)$

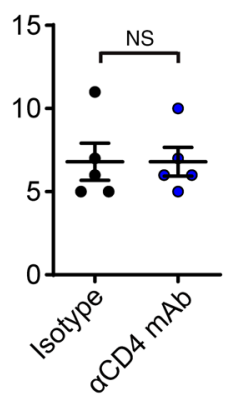

D

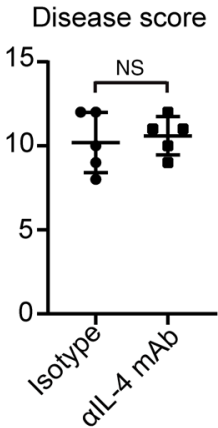

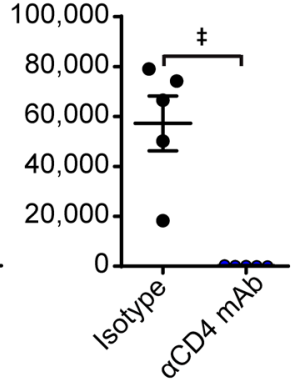

Serum $\lg E(\mathrm{ng} / \mathrm{mL})$

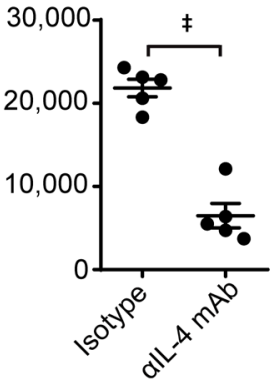

B Disease score Serum $\lg E(\mathrm{ng} / \mathrm{mL})$
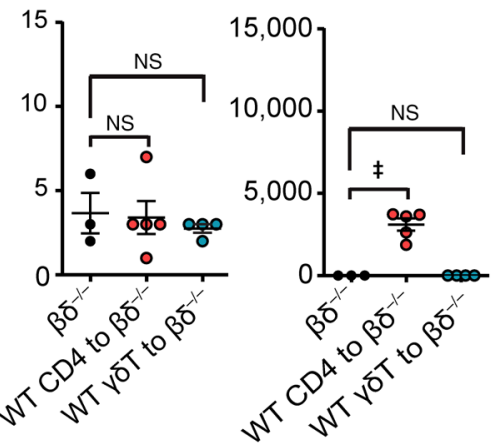

C Disease score

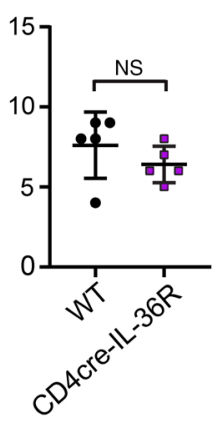

Serum $\lg E(\mathrm{ng} / \mathrm{mL})$

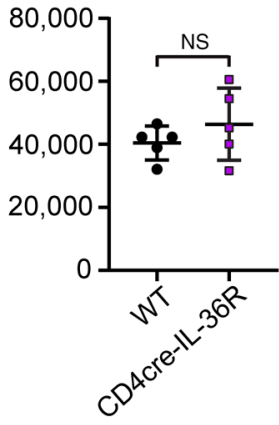

E
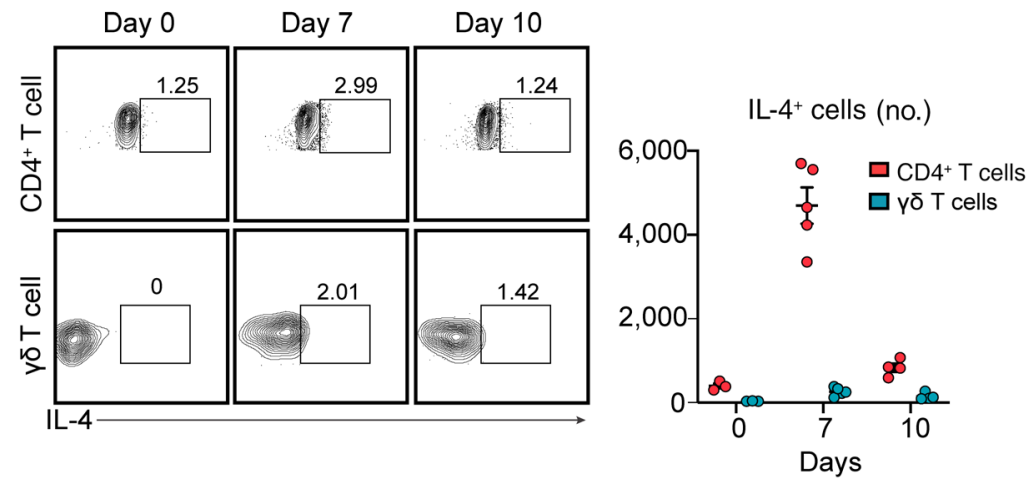

Figure 3. IL-36R-induced serum IgE levels are dependent on CD4+ $T$ cells and IL-4 in a mechanism independent of IL-36R-intrinsic signaling in CD4+ $T$ cells. (A-E) The S. aureus e.c. model was performed. (A) Mean disease score \pm SEM and mean total serum IgE \pm SEM after treating WT mice with anti-CD4 (to deplete $\mathrm{CD}^{+}$T cells) or isotype control mAb ( $n=5 /$ group). (B) $\beta \delta^{-/-}$mice (deficient in $\alpha \beta$ and $\gamma \delta$ T cells) with or without adoptive transfer of CD4+ or $\gamma \delta$ T cells from dLNs of naive WT mice ( $n=3-5 /$ group). (C) WT and CD4-cre $\times$ IL-36R ${ }^{\text {fl/fl }}$ mice (IL-36R deleted in CD4+ cells) ( $n=5 /$ group). (D) Mice treated with anti-IL-4 or isotype control mAb ( $n=5$ /group). (E) Representative flow plots and mean percentage of IL-4+ T cells (CD4+ versus TCR $\left.\gamma \delta^{+}\right) \pm$SEM from day 0 , 7, and $10 \mathrm{dLNs}$ of WT mice ( $n=4-5$ /group). ${ }^{\ddagger} P<0.001$ between indicated groups, as calculated by a 2 -tailed Student's $t$ test (A and $\mathbf{C}$-E) or 1-way ANOVA with Tukey correction for multiple comparisons (B). Results are representative of 2 independent experiments.

that IL-36R activity in part contributed to the induction of IL33 and TSLP transcripts in vivo. To directly evaluate whether IL-33 or TSLP contributed to skin inflammation and IgE production in the $S$. aureus e.c. model, skin disease score and serum IgE levels on day 10 were evaluated in WT and IL-33/- mice (Supplemental Figure 3, E and F) and in WT mice treated with an anti-TSLP neutralizing mAb ( $\alpha$ TSLP mAb) or isotype control antibody (Isotype, Supplemental Figure 3, G and H), as previously described (28). IL-33 $3^{--}$mice or mice treated with $\alpha$ TSLP had no significant differences in $\mathrm{AD}$-like skin inflammation as measured by disease score (Supplemental Figure 3, E and G) and serum IgE levels (Supplemental Figure 3, F and H), compared with control mice. Therefore, unlike results in IL-36R $\mathrm{R}^{-/}$mice (Figure 1, B and D), IL-33 and TSLP did not play a major role in contributing to skin inflammation and serum IgE levels in the $S$. aureus e.c. model.

Increased serum IgE in mice requires $C D 4^{+} T$ cells and $I L-4$ but not $T$ cell-intrinsic IL-36R signaling. The contribution of Th2 cells and $\operatorname{IL}-4(5,29)$ in $\operatorname{IgE}$ production were evaluated in the $S$. aureus e.c. model. First, WT mice were treated with an anti-CD4-depleting $\mathrm{mAb}$, which did not impact the increased AD-like skin inflammation as determined by disease score but blocked the induction of any serum IgE levels (Figure 3A). Second, adoptive transfer of $\mathrm{CD} 4^{+} \mathrm{T}$ cells from naive WT mice to $\alpha \beta$ and $\gamma \delta$ T cell-deficient mice $\left(\beta \delta^{-/-}\right.$mice) also did not induce increased skin inflammation (which was previously found to be dependent on both $\alpha \beta$ and $\gamma \delta \mathrm{T}$ cells, ref. 20) as determined by disease score but partially restored serum IgE induction (Figure $3 \mathrm{~B}$ ). This further demonstrates that the presence of $\mathrm{CD} 4^{+} \mathrm{T}$ cells was required to induce IgE production in a mechanism independent of an effect on skin inflammation. Third, newly generated cre/lox mice with IL-36R deleted in CD4 ${ }^{+}$ $\mathrm{T}$ cells (CD4-cre $\times$ IL-36 $\mathrm{R}^{\mathrm{fl} / \mathrm{fl}}$ mice) and WT mice had the same degree of increased skin inflammation as determined by the disease score and increased serum IgE levels (Figure 3C), indicating that the skin inflammation and $\operatorname{IgE}$ induction were independent of $\mathrm{CD}^{+} \mathrm{T}$ cell-intrinsic IL-36R signaling. Next, WT mice were treated with an anti-IL-4-blocking $\mathrm{mAb}$, which did not impact the increased skin inflammation as measured by the disease score but inhibited the induction of serum IgE (Figure 3D). Finally, intracellular flow cytometry of skin-draining lymph nodes (dLNs) revealed that $\mathrm{CD} 4^{+} \mathrm{T}$ cells (i.e., Th2 cells) were a more predominant $\mathrm{T}$ cell source of IL-4 compared with $\gamma \delta$ T cells (Figure $3 \mathrm{E}$ ), which is relevant because $\gamma \delta$ T cells have previously been shown to promote host defense in the $S$. aureus i.d. model $(23,24)$. It is also possible and likely that other cell types contributed to IL-4 production in the $S$. aureus e.c. model, such as ILC2s, basophils, eosinophils, and mast cells, which are all involved in the pathogenesis of AD (2-4). 
A
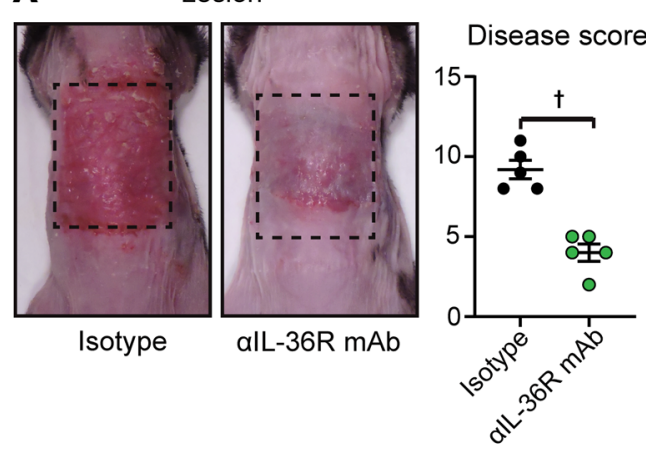

B

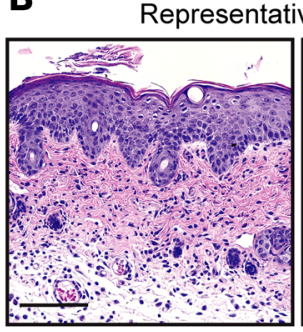

Isotype
E
D

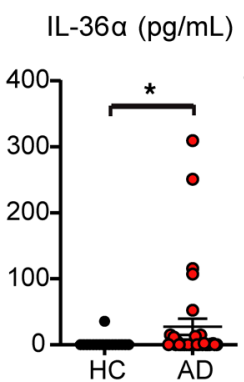

IL-36y (pg/mL)

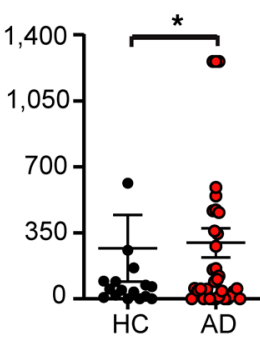

Serum IgE $(I U / \mathrm{mL})$

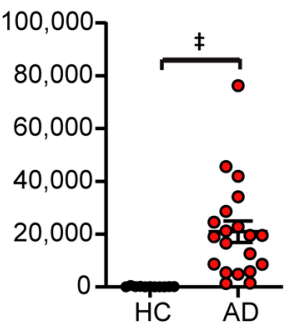

$\mathbf{F}$
C

Serum $\lg E(\mathrm{ng} / \mathrm{mL})$

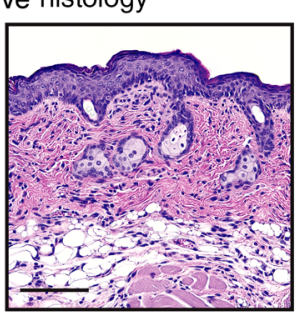

alL-36R mAb

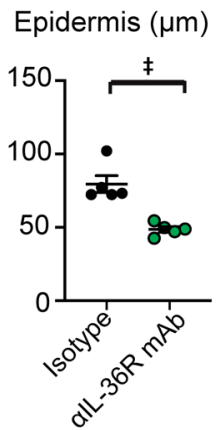

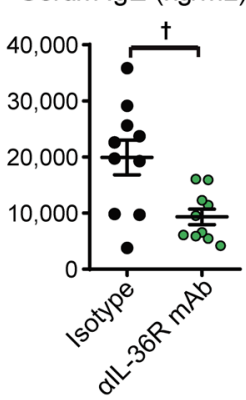

IL-36y
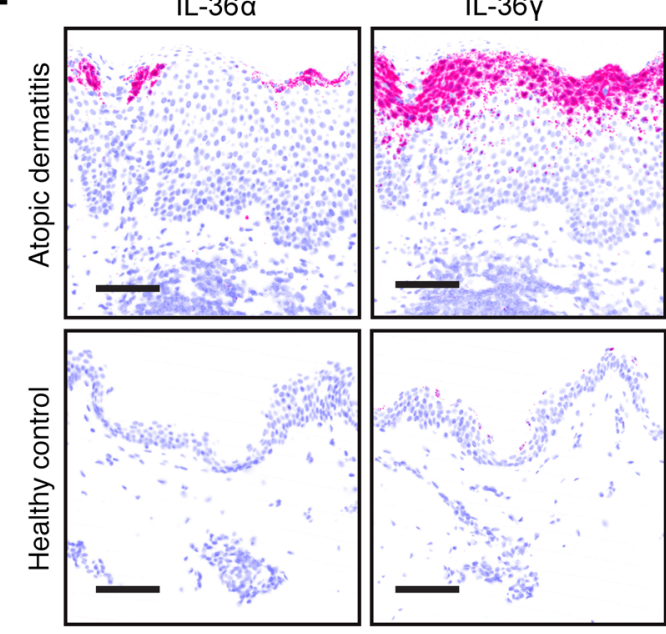

Figure 4. Antibody blockade of IL-36R inhibits skin inflammation and increased serum IgE levels in mice. (A-C) The S. aureus e.c. model was performed in WT mice treated with anti-IL-36R or isotype control mAb. (A) Representative skin photographs and mean disease score \pm SEM ( $n=5 /$ group). (B) Representative histology (H\&E stain; scale bars: $100 \mu \mathrm{m})$ and mean epidermal thickness $(\mu \mathrm{m}) \pm \operatorname{SEM}(n=5 /$ group $)$. (C) Mean serum IgE $(n g / m L) \pm S E M(n=10 /$ group). (D-F) Healthy HCs and patients with AD were evaluated for: (D) mean serum IL-36 $\alpha$ and IL-36 $\gamma$ protein levels (pg/mL) \pm SEM ( $n=14-29 / \mathrm{group}$ ); (E) mean total serum IgE (IU/mL) \pm SEM ( $n=14-29$ /group); and (F) representative human skin mRNA expression of $/ 136 \alpha$ and $/ I 36 \gamma$ by in situ hybridization (ISH). Scale bars: $100 \mu \mathrm{m} .{ }^{*} P<0.05,{ }^{\dagger} P<0.01,{ }^{\ddagger} P<0.001$ between indicated groups, as calculated by a 2-tailed Student's $t$ test. Results are representative of 2 independent experiments.

Nonetheless, our findings suggested that $\mathrm{CD}^{+} \mathrm{T}$ cells and IL- 4 are required for increased serum IgE levels independently of $\mathrm{T}$ cellintrinsic IL-36R signaling.

Anti-IL-36R-blocking antibody treatment inhibits skin inflammation and increased serum IgE levels in mice. IL-36 $\mathrm{R}^{-/-}$mice have constitutive global deletion of IL-36R and therefore it is possible that they might have developmental defects that could impair the ability of these mice to produce IgE. Therefore, to determine whether a developmental defect caused the lack of IgE induction in $\mathrm{IL}^{-36 \mathrm{R}^{-/}}$mice, WT mice were treated with an anti-IL-36Rblocking mAb. Similar to IL-36R $\mathrm{R}^{-/}$mice, the anti-IL-36R-blocking $\mathrm{mAb}$ resulted in decreased disease score, epidermal thickness, and serum IgE (Figure 4, A-C). This result raises the possibility that inhibiting IL-36R signaling might provide a potential therapeutic mechanism to block IgE production and ensuing IgEmediated allergic diseases in patients with $\mathrm{AD}$ who have markedly increased $S$. aureus skin colonization.

$I L-36$ cytokines in patients with AD and unopposed IL-36 activity in patients with human $I L-36$ receptor antagonist deficiency coincide with increased serum IgE. In translating these findings to humans, patients with AD had increased serum IL-36 $\alpha$ and IL-36 $\gamma$ levels (as reported previously by other groups, refs. 30-32) and increased

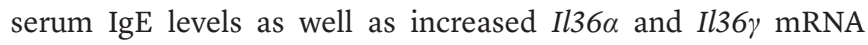
expression by in situ hybridization (ISH) in epidermal keratinocytes in their affected skin, compared with healthy controls (HCs) (Figure 4, D-F and Supplemental Figure 4). These results suggested that the elevated IL-36 cytokines in the serum and skin of AD patients could have contributed to the increased serum IgE levels that are often seen in $\mathrm{AD}$. Of note, there was no significant correlation between serum IL-36 $\alpha$ or IL-36 $\gamma$ protein levels and serum IgE levels at this single time point (data not shown). Furthermore, multiple patients with deficiency of interleukin thirty-six receptor antagonist (DITRA), who have unopposed IL-36 cytokine activity due to mutations in the IL-36 receptor antagonist $(I L-36 R N)$ and develop generalized pustular psoriasis (GPP), had increased serum IgE (445 IU/mL [ref. 33], $1641 \mathrm{IU} / \mathrm{mL}$ [ref. 34], and $1058 \mathrm{IU} /$ $\mathrm{mL}$ [patient in the present study]; normal range: 0-100 IU/mL). Although patients with DITRA do not develop AD-like skin inflammation but rather develop epidermal pustules and psoriatic-like skin inflammation in GPP, these data provide an explanation for how unopposed IL-36 activity in patients with DITRA might have similarly contributed to the observed increased serum IgE levels. 
A

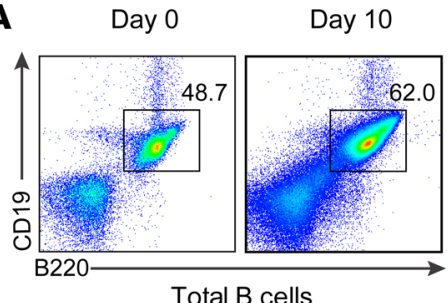

C

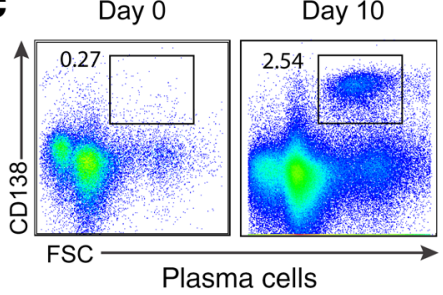

B

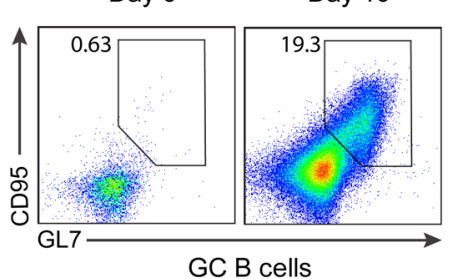

D

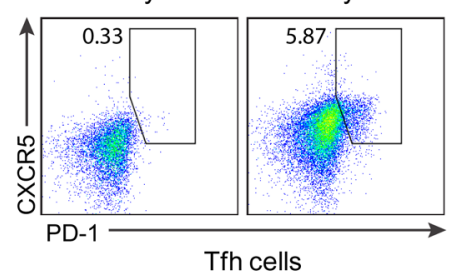

E

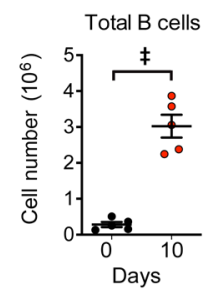

$\mathbf{F}$

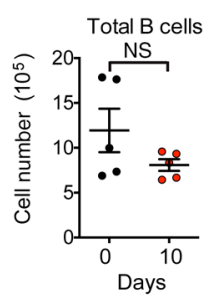

G

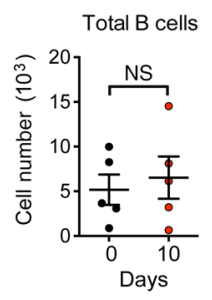

Lymph nodes
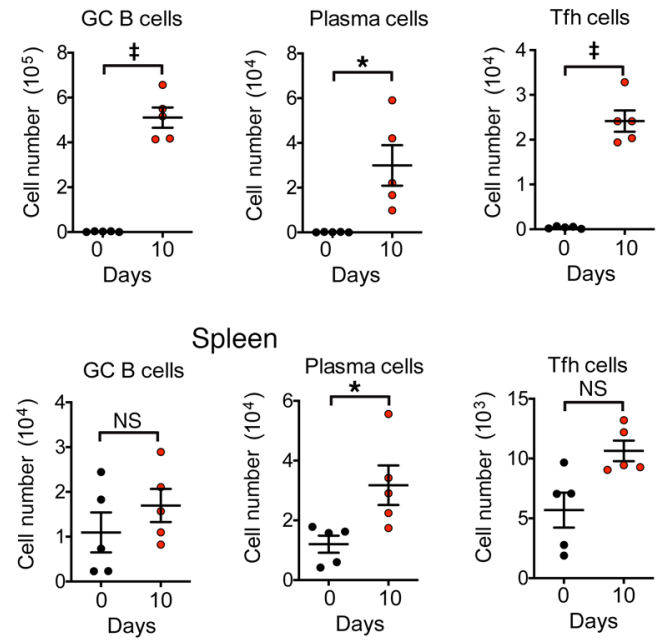

Spleen
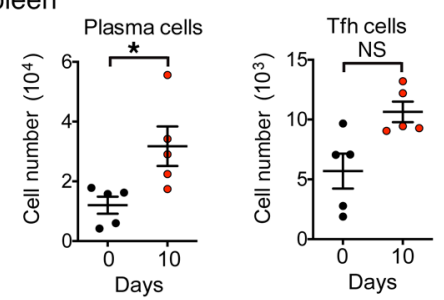

Peyer's patches
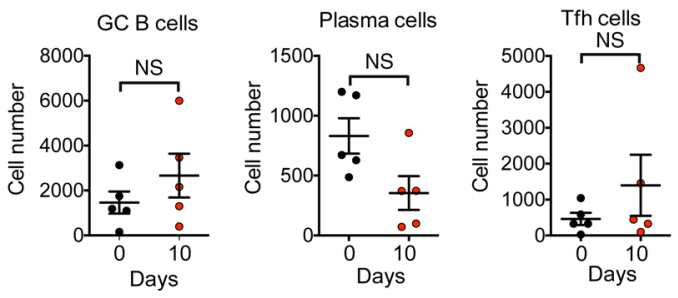

Figure 5. Numbers of total B cells, GC B cells, PCs, and Tfh cells following the $\boldsymbol{S}$. aureus e.c. model. dLNs, spleens, and Peyer's patches were isolated on day 0 (naive WT mice) and day 10 following the S. aureus e.c. model in WT mice ( $n=5$ /group). (A-D) Representative flow plots from cells isolated from dLNs for B cells (CD19+B220+ cells) (A), GC B cells (CD95 ${ }^{+}$CL7 $7^{+}$cells) (B), PCs (CD138+FSChi), (C) and Tfh cells (CXCR5+PD-1+ cells) (D). (E-C) Mean cell number \pm SEM in the dLNs $(\mathbf{E})$, spleens $(\mathbf{F})$, and Peyer's patches (G). ${ }^{*} P<0.05,{ }^{\ddagger} P<0.001$ between indicated groups, as calculated by a 2-tailed Student's $t$ test. Results are representative of 2 independent experiments.

but not GC B cells were decreased, compared with WT mice (Figure 6, A and B). IL-1 $\beta^{-/-}$mice were also evaluated as a comparison group (as in Supplemental Figure 1, $\mathrm{B}-\mathrm{D}$ ) and no differences in total or $\mathrm{IgE}^{+} \mathrm{GC}$ B cells or PCs between IL-1 $1 \beta^{--}$and WT mice were observed, indicating the specificity to IL-36R signaling (Supplemental Figure 6). Transcripts involved in class-switching (Aicda and Il4ra), PC differentiation (Blimp1 and Xbp1; Figure 6C), B cell activation, $\mathrm{GC}$ formation (Bcl6, Mcl1, and $I d 2), \mathrm{T}$ cell help (IL21 and Cd4Olg), hyper-IgE syndromes (Stat3 and $Z f p 341$ ), and other relevant transcripts (IL9 and IL36r) (35-40) were evaluated in dLNs on days 0 and 10 from WT and IL-36R $\mathrm{R}^{-/}$mice (Supplemental Figure 7). Aicda, Blimp1, and Xbp1 were decreased in IL-36 $\mathrm{R}^{-/-}$versus WT mice with no other differences observed, including IL4ra (which would increase B cell responsiveness to IL-4) and IL9 (which promotes IgE production and is

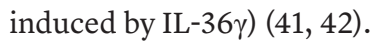

$I L-36 \alpha$ enhances IL-4-mediated B cell production of IgE in vitro. To evaluate whether IL-36 $\alpha$ could enhance IL-4-mediated IgE production, naive B cells from dLNs and spleens of WT mice were cultured in antibody-producing conditions with or without rIL-4 or rIL-4+rIL-36 $\alpha$ (Figure 7, A-C). rIL-4+rIL-36 $\alpha$ induced nearly twice the number of $\operatorname{IgE}^{+}$ELISpots than rIL-4 (Figure 7A), with no difference in $\mathrm{B}$ cell proliferation (Figure 7B). B cells from $\mathrm{IL}-36 \mathrm{R}^{-/}$mice were also evaluated and the number of $\mathrm{IgE}^{+}$ELISpots induced by rIL-4 and rIL-36 $\alpha+$ rIL-4 were not different (Supplemental Figure 8), indicating that the increase in $\mathrm{IgE}^{+}$ELISpots required $\mathrm{B}$ cell-intrinsic IL-36R signaling. The transcripts in Figure 7C and Sup-

$d L N$ s in mice are the source of increased IgE-producing B cells and PCs. To evaluate a role for B cell-intrinsic IL-36R signaling, dLNs, spleens, and Peyer's patches from days 0 and 10 of the $S$. aureus e.c. model in WT mice were evaluated (Figure 5). dLNs had increased total B cells $\left(\mathrm{CD} 19^{+} \mathrm{B} 22 \mathrm{O}^{+}\right)$, germinal center $(\mathrm{GC})$ B cells $\left(\mathrm{CD}^{+} 5^{+} \mathrm{GL}^{+}\right)$, PCs $\left(\mathrm{CD} 138^{+}\right.$forward scatter $\left.[\mathrm{FSC}]^{\mathrm{h}}\right)$, and $\mathrm{T}$ follicular helper (Tfh) cells (CD4 ${ }^{+} \mathrm{CXCR}^{+} \mathrm{PD}-1^{+}$; Figure 5, A-E) whereas spleens had an increase only in PCs (Figure 5F). No cellular changes were observed in Peyer's patches (Figure 5G). Thus, increased B cells and PCs predominantly occurred in dLNs. Also, there were no baseline differences in B cells, GC B cells, or PCs between naive WT (i.e., day 0, prior to performing the S. aureus e.c. model) and IL-36 $\mathrm{R}^{-/-}$mice (Supplemental Figure 5). In IL-36 $\mathrm{R}^{-/-}$mice on day 10 , total GC B cells and PCs and IgE $\mathrm{ICs}^{+}$ plemental Figure 6 were evaluated and rIL-36 $\alpha+$ rIL- 4 resulted in increased Xbp1 and decreased Bcl6 and Id 2 compared with rIL-4, with no other differences (Figure 7C and Supplemental Figure 9).

IL-36R signaling promotes skin allergen sensitization and subsequent allergic lung inflammation in mice. To determine whether IL-36R signaling contributed to the progression from AD-like skin inflammation to allergic lung inflammation, cockroach antigen (CrA) skin allergen sensitization (CrA is a clinically relevant allergen for AD and allergic asthma, refs. 43, 44) or PBS (negative control) with or without the S. aureus e.c. model was performed in WT mice for 7 days followed by all of the mice in these experiments undergoing an intratracheal (i.t.) challenge with $\mathrm{CrA}$ (days 10, 11, and 12; Figure $8 \mathrm{~A}$ ). Lung inflammation, bronchoalveolar lavage (BAL) fluid, neutrophil, eosinophil, and basophil numbers, and chang- 
A

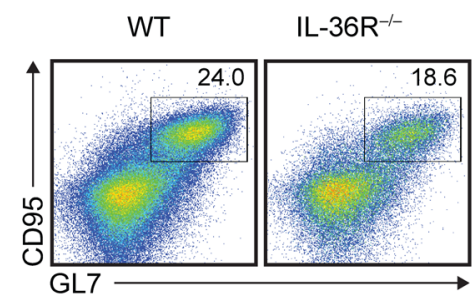

B

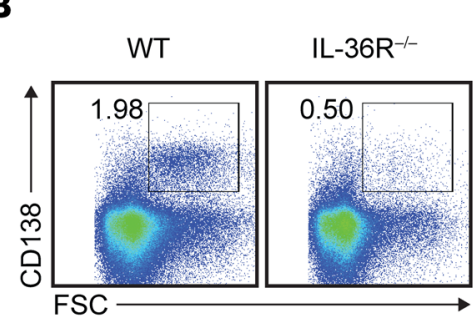

C

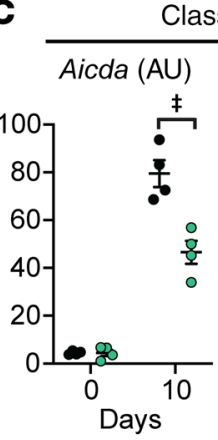

GC B cells (no.)

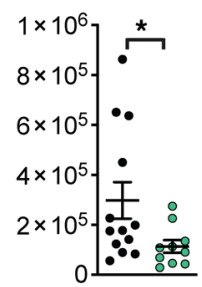

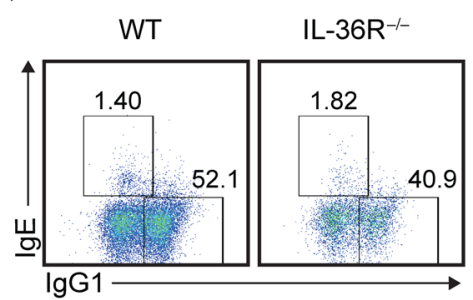

PCs (no.)
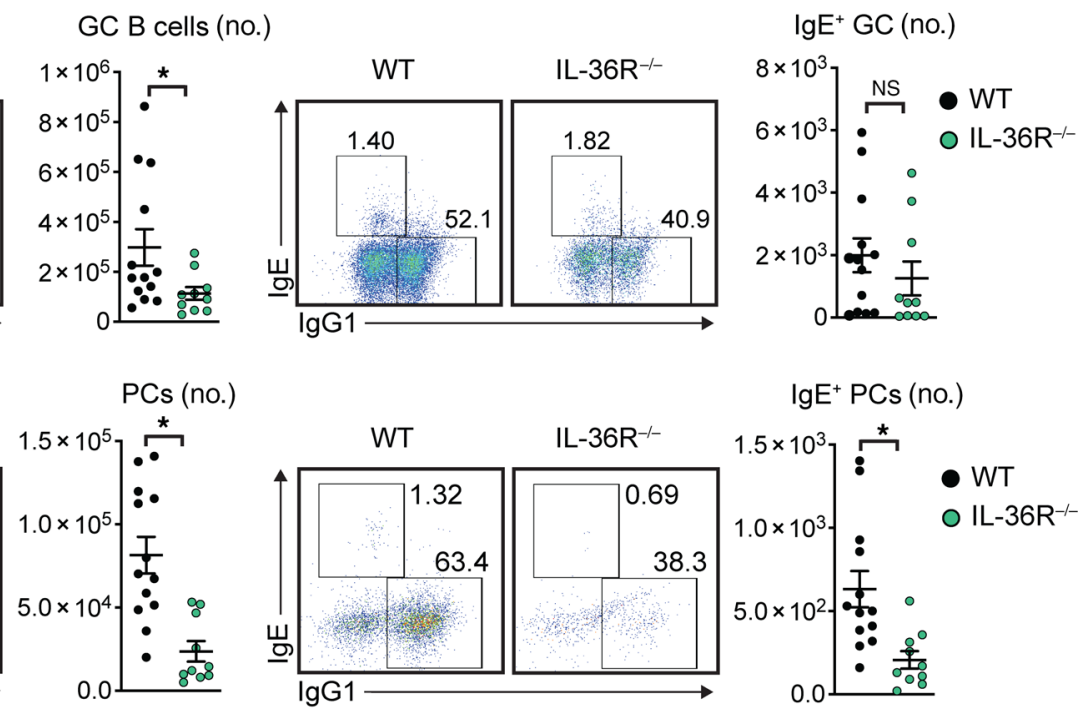

$\lg \mathrm{E}^{+} \mathrm{PCs}(\mathrm{no})$

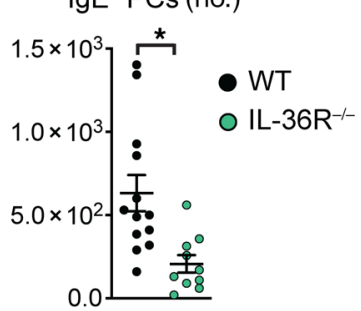

Plasma cell differentiation
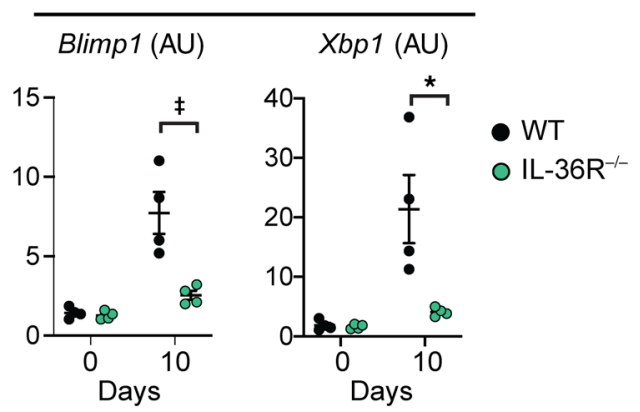

Figure 6. IL-36R signaling increases B cell class-switching, PC numbers, and IgE production in vivo. (A-C) The S. aureus e.c. model was performed in WT and IL-36R $\mathrm{R}^{-1-}$ mice and the following were evaluated from dLNs. (A and B) Representative flow plots and mean cell number \pm SEM of total and IgE ${ }^{+} \mathrm{GC} B$ cells (CD95 $\left.{ }^{+} \mathrm{CL7}^{+}\right)\left(n=10\right.$ /group) (A) and PCs (CD138 ${ }^{+} \mathrm{FSC}$ hi) ( $n=10$ /group) on day 10 (B). (C) Mean mRNA levels (AU) \pm SEM of transcripts for Aicda, $/ 14$ ra, Blimp1, and $X b p 1$ on days 0 and 10 ( $n=4$ /group). ${ }^{*} P<0.05,{ }^{\ddagger} P<0.001$ between indicated groups, as calculated by a 2-tailed Student's $t$ test. Results are representative of 2 independent experiments.

es in body weights and serum IgE levels were evaluated (Figure 8, B-F and Supplemental Figure 10). S. aureus e.c. alone and PBS had no differences in lung inflammation, BAL neutrophil numbers, or body weights, despite $S$. aureus e.c. inducing IgE (Figure 8, B-F). As expected, e.c. CrA alone had moderately increased lung inflammation, no difference in BAL neutrophil numbers or weight change, and no IgE induction (Figure 8, B-F). In contrast, in WT mice, e.c. S. aureus with $\mathrm{CrA}(\mathrm{SA}+\mathrm{CrA})$ resulted in marked lung inflammation, increased BAL neutrophil numbers, decreased body weights, and increased IgE (Figure 8, B-F). Importantly, all of these responses were markedly attenuated in IL-36R $\mathrm{R}^{-/}$mice, demonstrating that the enhanced allergic lung inflammation following e.c. $\mathrm{SA}+\mathrm{CrA}$ was dependent on IL-36R signaling. Disease scores were as expected with e.c. SA alone and e.c. SA+CrA having increased disease scores compared with PBS, e.c. CrA or e.c. SA+CrA in IL-36 $\mathrm{R}^{-/}$mice (Figure 9A). Furthermore, no significant differences in BAL eosinophil and basophil numbers were found between e.c. CrA alone and SA+ CrA mice or between SA+CrA-treated WT and IL-36R $\mathrm{R}^{-/}$mice (Supplemental Figure 10), suggesting that the number of neutrophils was a more important factor in distinguishing the cellular differences involved in this model of allergic lung inflammation.
Skin allergen sensitization during the S. aureus e.c. exposure was required for enhancing allergic lung inflammation in mice. Finally, if e.c. SA+CrA was followed by i.t. ovalbumin (OVA) (without prior OVA skin sensitization), there were no differences in lung inflammation, BAL neutrophil numbers, or weight change compared with no i.t. challenge (Figure 9, B-E). This result provides additional evidence that the prior skin allergen sensitization along with the concomitant $S$. aureus e.c. exposure was required for the enhanced allergic lung inflammation. In combining the results in Supplemental Figures 8 and 9, a mechanism is uncovered in which skin allergen sensitization in the setting of $S$. aureus e.c. exposure, as occurs in the defective epidermal barrier and dysbiosis in the skin of patients with $\mathrm{AD}$, leads to IL-36-dependent increased serum IgE production and subsequent allergic lung inflammation.

\section{Discussion}

Type 2 immune responses in AD have been implicated in $\operatorname{IgE}$ production and progression to allergic diseases, but whether keratinocytes in $\mathrm{AD}$ skin can directly trigger $\mathrm{B}$ cell and $\mathrm{PC}$ IgE production is unclear. In a mouse model of AD-like skin inflammation in response to $S$. aureus e.c. exposure, there were substan- 
A

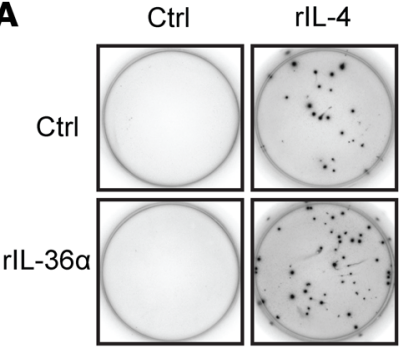

lgE ELISpots (no.)

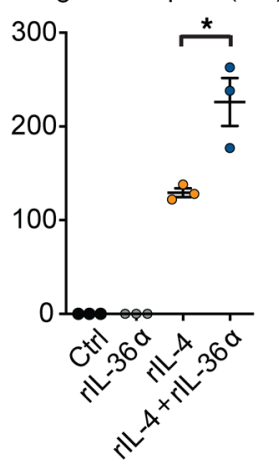

B

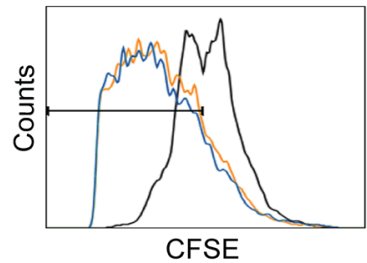

Proliferation (\%)

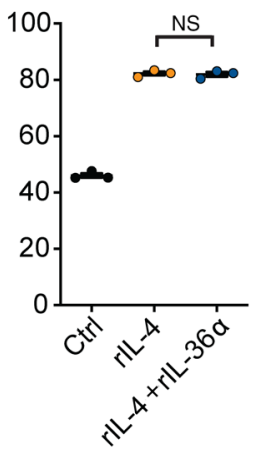

C

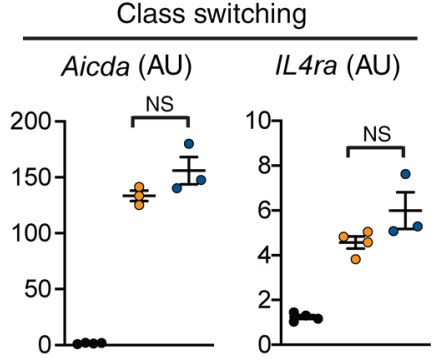

Plasma cell differentiation

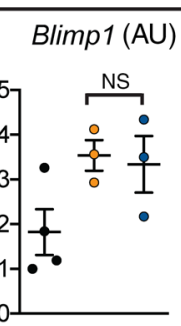

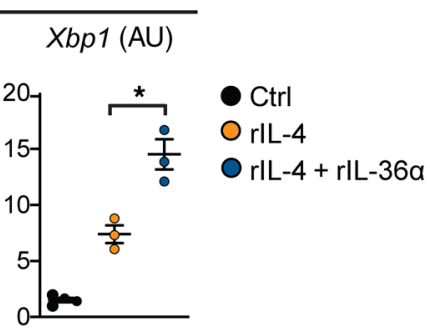

Figure 7. IL-36 $\alpha$ enhances IL-4-mediated IgE production and PC differentiation in B cells. Naive B cells purified from dLNs and spleens of WT mice were cultured for 5 days \pm rlL-4 or rlL-4+rlL-36 $\left(n=3\right.$ /group) and the following were measured. (A) Representative IgE $E^{+}$ELISpots and mean number of IgE ${ }^{+}$ ELISpots \pm SEM. (B) Representative histogram of CFSE fluorescence and mean percentage \pm SEM of in vitro B cell proliferation. (C) Mean mRNA levels (AU) \pm SEM of transcripts for Aicda and IL-4ra (involved in class-switching) and Blimp1 and Xbp1 (involved in PC differentiation). ${ }^{*} P<0.05$, between indicated groups, as calculated by a 2-tailed Student's $t$ test. Results are representative of 2 independent experiments.

tially elevated serum IgE levels, which, beyond Th2 cell and IL-4 responses, unexpectedly required IL-36R signaling. Activation of IL-36R signaling was initiated by keratinocyte-derived IL-36 $\alpha$ in the inflamed skin in vivo that directly activated B cell and PC class-switching to IgE production in vitro. Moreover, IL-36R signaling was also essential for the progression from skin allergen sensitization during $S$. aureus e.c. exposure-induced AD-like inflammation to subsequent allergic lung inflammation. Taken together, these findings provide several new and important insights into the pathogenesis of allergic diseases.

In $\mathrm{AD}$, it is thought that keratinocyte-derived IL-33 $(12,13)$ and TSLP (14-16) contribute to type 2 immune responses that mediate IL-4-dependent IgE production and subsequent development of other allergic diseases (2-4). However, we found that IL-36 $\alpha$ produced in the inflamed skin following $S$. aureus e.c. exposure has direct functional activity in triggering $B$ cell production of IgE (beyond the activity of IL-4) and differs from the activity of IL-33 and TSLP, which promote early B cell development $(17,18)$. Although we demonstrate that Th2 cells were a more predominant T cell source of IL-4 than $\gamma \delta \mathrm{T}$ cells, many other cells likely contributed to the production of IL-4 in the $S$. aureus e.c. model, including ILC2s, basophils, eosinophils, and mast cells involved in $\mathrm{AD}$ pathogenesis (2-4). In addition, we found that $S$. aureus induces Il33 and Tslp transcript levels in mouse keratinocytes in vitro (Supplemental Figure 3, A and B), consistent with prior reports $(26,27)$, and in the $S$. aureus e.c. model in vivo in a mechanism partially dependent on IL-36R activity (Supplemental Figure 3, C and D). Moreover, IL-33-/- mice or mice treated with
aTSLP had no significant differences in induction of AD-like skin inflammation or serum IgE levels (Supplemental Figure 3, E-H), suggesting that both increased skin inflammation and serum IgE levels were induced to a greater degree by IL-36R activity rather than by IL-33 or TSLP activity. These results do not preclude possible synergistic effects among IL-36 cytokines, IL-33, and/or TSLP in contributing to type 2 immune responses, as IL-33 and TSLP can skew toward type 2 immune responses in the pathogenesis of $\mathrm{AD}(2-4)$. The potential synergistic, interactive, and/or differential effects among IL-36 cytokines, IL-33, and TSLP will be the subject of our future work.

The IL-36R response in inducing IgE required $\mathrm{CD}^{+} \mathrm{T}$ cells, as IgE production was abrogated in mice treated with an anti$\mathrm{CD} 4$-depleting $\mathrm{mAb}$ and serum IgE levels were partially restored following the adoptive transfer of $\mathrm{CD}^{+} \mathrm{T}$ cells from WT mice into $\beta \delta^{-/-}$mice. Also, the ability of IL-36 cytokines to directly induce AD-like skin inflammation in the $S$. aureus e.c. mouse model (such as by directly activating IL-36R expressed by keratinocytes) was not investigated in this initial report and will be the focus of our future work. Furthermore, the IL-36R response did not involve $\mathrm{CD}^{+} \mathrm{T}$ cell-intrinsic IL-36R signaling because there were no differences in the increased $\mathrm{AD}$-like skin inflammation or $\operatorname{IgE}$ production induced during the $S$. aureus e.c. model in CD4-cre $\times$ $\mathrm{IL}-36 \mathrm{R}^{\mathrm{fl} / \mathrm{fl}}$ mice compared with WT mice. Thus, the IgE production that was dependent on $\mathrm{CD} 4^{+} \mathrm{T}$ cells occurred in a mechanism that was independent of any effects on the AD-like skin inflammation.

In our prior work (20), we found that adoptive transfer of pan-T cells (i.e., both $\alpha \beta$ and $\gamma \delta$ T cells) from WT mice into IL-36R- 
A

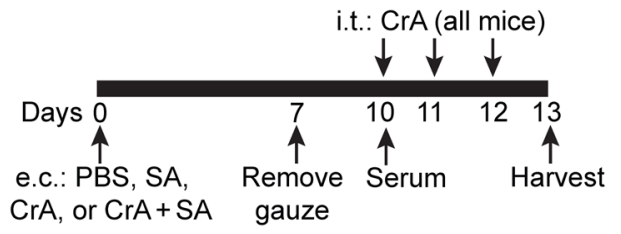

B
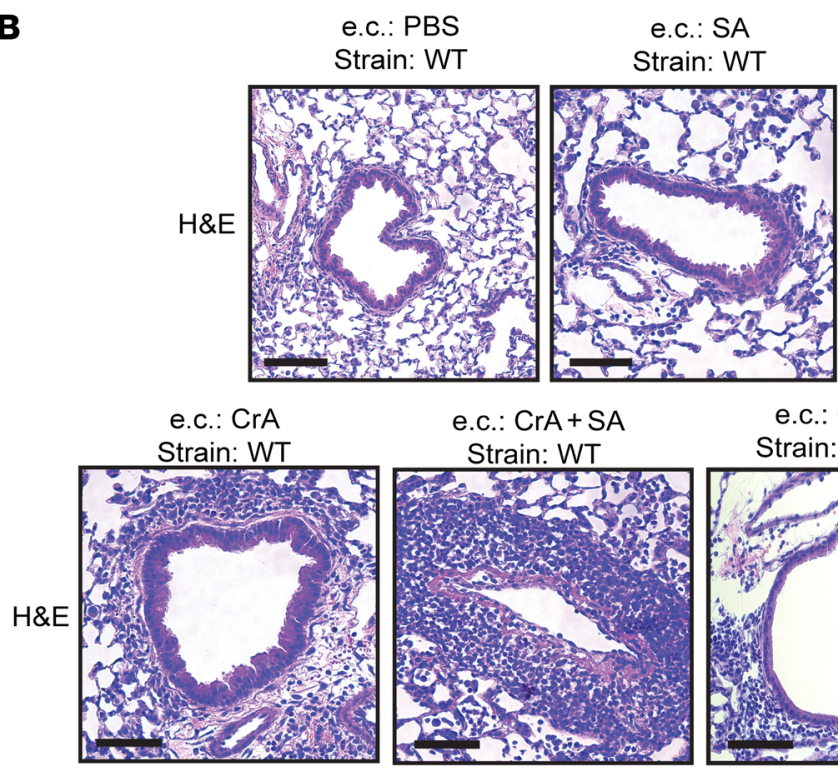

e.c.: $\mathrm{CrA}+\mathrm{SA}$

Strain: IL-36R-1-
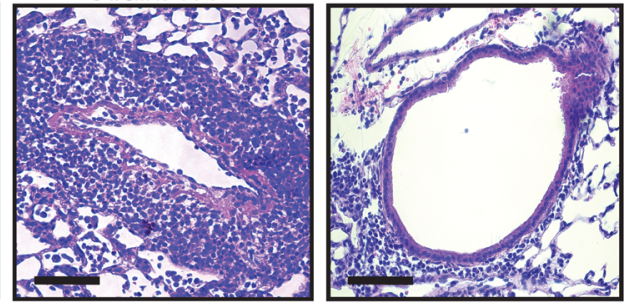

\section{Lung inflammation (\%)}

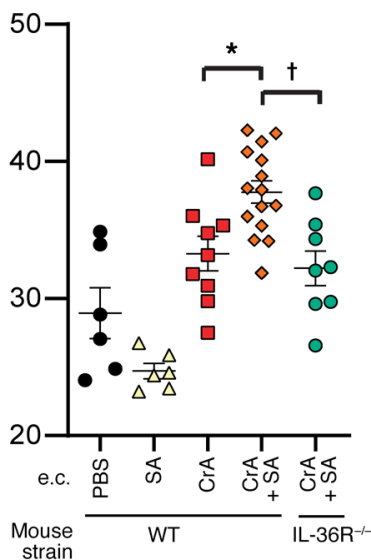

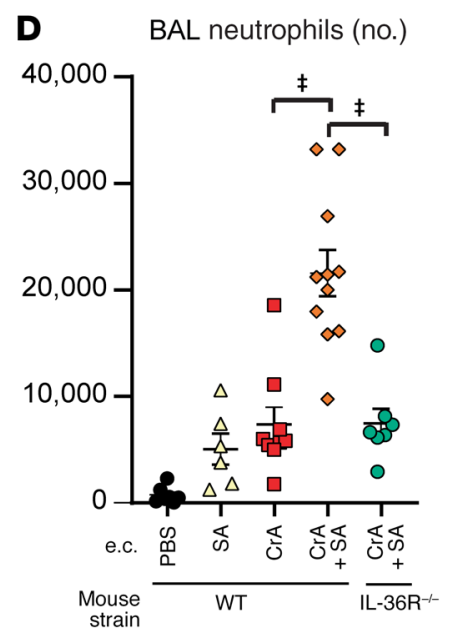

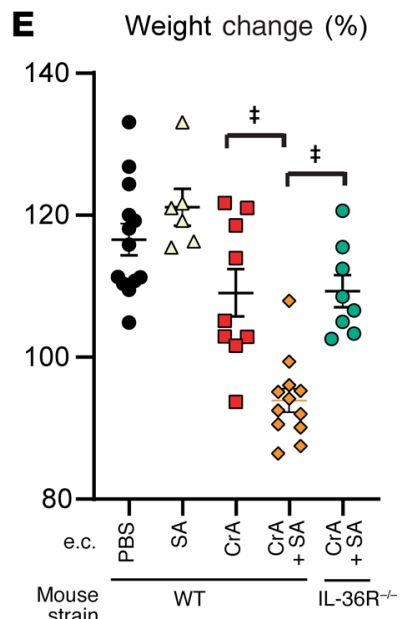

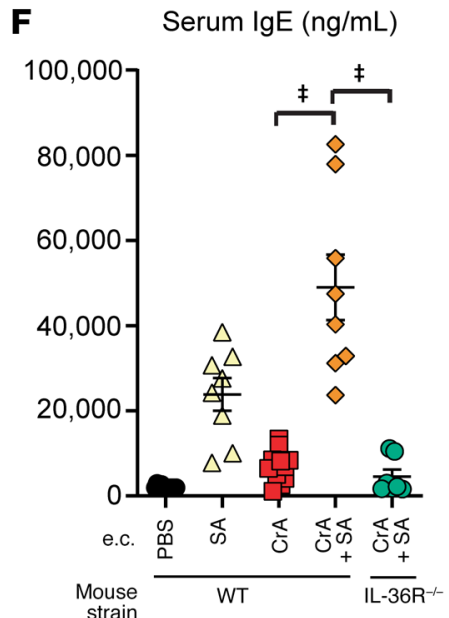

Figure 8. IL-36R signaling promotes skin allergen sensitization and subsequent allergic lung inflammation associated with increased serum IgE. (A) Timeline of e.c. challenge of WT and IL-36R ${ }^{-1-}$ mice with PBS, S. aureus (SA) alone, cockroach antigen (CrA) alone, or SA+CrA combined ( $\left.n=6-13 / g r o u p\right)$ to the skin for 7 days (as in the S. aureus e.c. model in Figure 1A). On days 10, 11, and 12, the same mice were challenged i.t. with $\mathrm{CrA}$, serum was harvested on day 10, and lung tissue and BAL fluid were harvested on day 13. (B) Representative lung histology (H\&E stain; scale bars: $100 \mu \mathrm{m}$ ). (C) Lung inflammation from histologic sections as measured by Image J software analysis as the percentage of the density of H\&E staining \pm SEM. (D) Mean neutrophil number in the BAL fluid \pm SEM, as measured by FACS. (E) Percentage of body weight change from days 0 to $13 \pm S E M$. (F) Mean total serum IgE (ng/mL) \pm SEM. ${ }^{*} P<0.05,{ }^{\dagger} P<0.01,{ }^{\ddagger} P<0.001$ between indicated groups, as calculated by 1-way ANOVA with the Tukey correction for multiple comparisons. Results are representative of 2 independent experiments.

deficient mice partially rescued the increased AD-like skin inflammation induced in the $S$. aureus e.c. model. Combining these previous results with the findings in the present study involving specific adoptive transfer of $\mathrm{CD}^{+} \mathrm{T}$ cells from WT mice to IL-36R-deficient mice (see Figure 3, A-C) and experiments with the CD4-cre $\times \mathrm{IL}^{-36 \mathrm{R}^{\mathrm{A} / \mathrm{l}}}$ mice, it can be inferred that non-CD4 ${ }^{+} \mathrm{T}$ cell subsets (e.g., $\mathrm{CD}^{+} \mathrm{T}$ cells, $\gamma \delta \mathrm{T}$ cells, and/or NK T cells) were predominantly involved in the induction of increased AD-like skin inflammation, whereas $\mathrm{CD} 4^{+} \mathrm{T}$ cells primarily mediated the increased serum IgE levels. Our future work will include generating and performing experiments with conditional knockout mouse strains by crossing different cre mice to the newly generated IL- $36 \mathrm{R}^{\mathrm{f} / \mathrm{f}}$ mice so that IL-36R is deleted in additional cell types, including B cells.
The increased serum IgE levels in the S. aureus e.c. model suggested that surface exposure of the keratinocytes to $S$. aureus might be required. This finding was demonstrated as increased serum IgE levels were only observed in the $S$. aureus e.c. exposure model but not when $S$. aureus was inoculated in an i.d. challenge model, which is likely due to the preferential and high induction of IL-36 $\alpha$ in the S. aureus e.c. model. The reason for the differences in the ensuing host response related to IL-36 responses and IgE production between the e.c. and i.d. bacterial exposure was most likely due to the anatomical location of the infection, as the host response at distinct anatomical sites of infection in the skin can be different, as was previously demonstrated in a wound versus i.d. S. aureus exposure that used the same bacterial biolumines- 


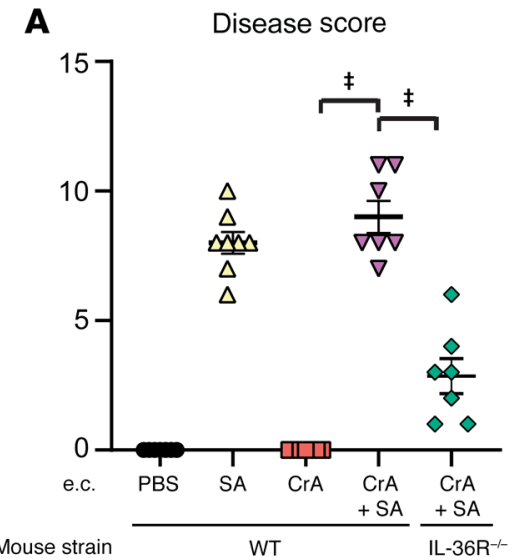

B

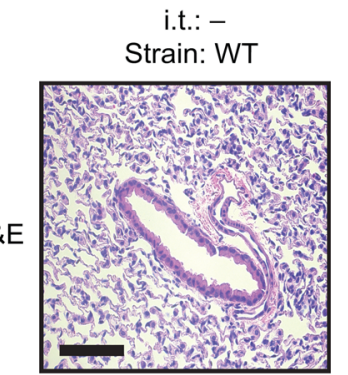

i.t.: OVA

Strain: WT

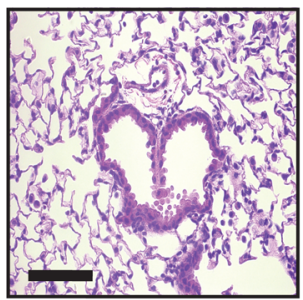

C Lung inflammation (\%)

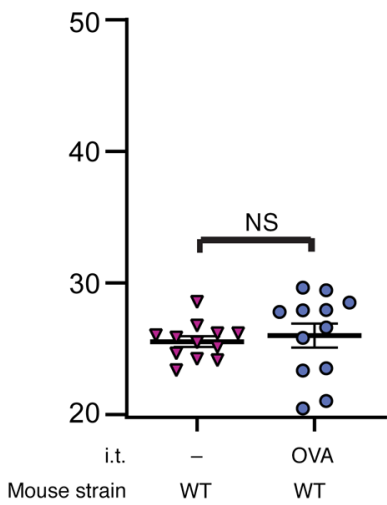

D BAL neutrophils (no.)

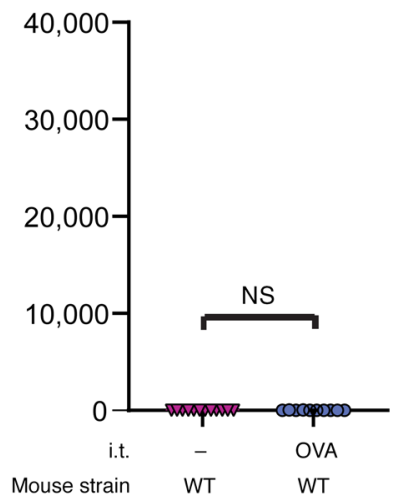

E Weight change (\%)

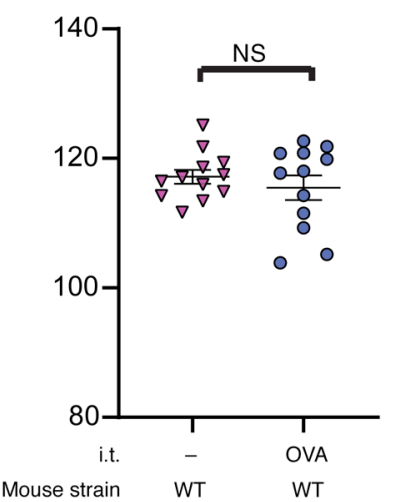

Figure 9. IL-36R signaling that promotes skin allergen sensitization and subsequent allergic lung inflammation is due to specific antigen sensitization in the skin. (A-E) The e.c. challenge of WT and IL-36R ${ }^{-1-}$ mice was performed with PBS, S. aureus (SA) alone, cockroach antigen (CrA) alone, or SA+CrA combined ( $n=6-10$ /group) to the skin for 7 days (as in Figure 8 ). On days 10,11 , and 12 , the same mice were either not challenged (-) or challenged intratracheally (i.t.) with CrA or OVA (as an alternative allergen to CrA that was not applied to the skin during the e.c. challenge). Lung tissue and BAL fluid were harvested on day 13. (A) Mean disease score \pm SEM ( $n=6-10 /$ group). (B) Representative lung histology (H\&E stain; scale bars: $100 \mu m$ ) without i.t. challenge (-) or following i.t. challenge with OVA ( $n=12 /$ group). (C) Lung inflammation from histologic sections as measured by Image J software analysis as the percentage of the density of H\&E-staining \pm SEM ( $n=12 /$ group). (D) Mean neutrophil number in the BAL fluid \pm SEM, as measured by FACS ( $n=12 /$ group). (E) Percentage of body weight change from days 0 to $13 \pm$ SEM ( $n=12$ /group). ${ }^{\ddagger} P<0.001$ between indicated groups, as calculated by a 1 -way ANOVA with the Tukey correction for multiple comparisons (A)or a 2-tailed Student's $t$ test (B-E). Results are representative of 2 independent experiments.

cent $S$. aureus strain (45). The anatomical site-specific differences between the e.c. versus i.d. models likely involves the enhanced epidermal inflammation in the e.c. model compared with the i.d. model. For example, in the S. aureus e.c. model, previous work indicated that the $S$. aureus-derived cytolytic toxins (i.e., $\delta$-toxin and phenyl soluble modulin alpha [PSM $\alpha]$ ) contributed to the $S$. aureusinduced skin inflammation (19-21). Whether $\delta$-toxin, PSM $\alpha$, or other $S$. aureus-derived toxins, superantigens, or proteases (and potentially other bacterial factors, refs. 9-11) contributed to the IL-36R-dependent increased IgE and ensuing allergic responses, and this will be the subject of our future work. In contrast, the S. aureus i.d. model results in the formation of a dermal neutrophilic abscess with overlying epidermal necrosis, which is not observed in the $S$. aureus e.c. model. In addition, other bacterial-specific differences could be involved such as different bacterial metabolic profiles and bacterial-derived factors produced on the surface of the skin in the e.c. model compared with the intradermal tissue and the neutrophilic abscess in the i.d. model. Finally, it could be that there were bacterial-specific differences between the $2 \mathrm{~S}$. aureus bioluminescent strains used separately in the e.c. and i.d. models, which had different bioluminescent lux constructs inserted into the same parental USA300 LAC $S$. aureus strain.

In translating our findings to humans, we found elevated serum IL-36 $\alpha$ and IL-36 levels along with increased serum IgE in patients with $\mathrm{AD}$ as well as increased Il36 $\alpha$ and Il36y expression in keratinocytes in histologic sections of inflamed $\mathrm{AD}$ skin, providing further insights into the previously observed increased $I l 36 \alpha$ and $I l 36 \gamma$ transcripts in $\mathrm{AD}$ skin (30-32). Although there was no significant correlation between serum IL-36 $\alpha$ or IL-36 $\gamma$ protein levels and serum IgE levels in patients with $\mathrm{AD}$ at the single time point assessed (Figure 4, D and E), this result does not necessarily indicate that IL-36 cytokines did not contribute to the increased serum IgE levels earlier in the course of this chronic and often lifelong disease. We further found that patients with DITRA (who have increased $I L-36 \alpha$ and $I L-36 \gamma$ levels, ref. 46) also had elevated serum IgE levels. Therefore, a link between IL-36 activity in the skin and increased serum IgE levels is supported by these findings in humans. Moreover, these findings provide an explanation for the 
increased serum IgE levels in patients with DITRA who have GPP, which is a type of skin inflammation that involves epidermal pustules and psoriasis-like skin inflammation rather than AD-like skin inflammation. Therefore, the unopposed IL-36 responses in the inflamed skin of patients with DITRA that is not associated with type 2 immune responses provide an explanation for the observed increased serum IgE levels in these patients. In addition, the generally lower levels of IgE in the patients with DITRA compared with the patients with $\mathrm{AD}$ were likely due to the additional effects of the type 2 immune responses (e.g., IL-4-mediated immune responses) in patients with AD that are not found in patients with DITRA. It should be mentioned that both IL-36 $\alpha$ and IL-36 levels were increased in patients with AD and DITRA, whereas only IL-36 $\alpha$ was found elevated in the $S$. aureus e.c. mouse model. The reason for this is not entirely clear, but could be due to intrinsic aspects of the $S$. aureus e.c. mouse model (which does not fully recapitulate all features of human $\mathrm{AD}$ ) or species-specific differences.

Prior work has found that in the context of autoimmune arthritis (i.e., rheumatoid and psoriatic arthritis), increased levels of IL-36 $\alpha$ were shown to activate B cells and PCs, which were suggested to contribute to synovial inflammation $(47,48)$. In addition, synovial PCs were identified as the primary source of IL-36 $\alpha$. In contrast, in the context of AD-like skin inflammation in the $S$. aureus e.c. model, IL-36 $\alpha$ directly activated B cells and PCs in dLNs to promote to class-switching and production of IgE, defining a direct functional consequence of IL-36 activity. Furthermore, while it is possible that PCs (or potentially other cells) were a cellular source of IL-36 $\alpha$ during the $S$. aureus e.c. model, increased IL-36 $\alpha$ transcript and protein levels were found in the keratinocytes in the inflamed skin in vivo, and only exogenously administered recombinant IL-36 $\alpha$ was able to enhance B cell production of IgE in vitro, suggesting that keratinocytes were the primary source of IL-36 $\alpha$ in the $S$. aureus e.c. model. IL-36 also resulted in specific transcriptional changes associated with $B$ cell function, including expression of Aicda, Blimp1, and Xbp1 in dLNs in vivo and increased $X b p 1$ and decreased Bcl6 and $I d 2$ expression in response to rIL-4+rIL-36 $\alpha$ in B cells in vitro. Curiously, the transcription factor $X b p 1$ involved in PC differentiation and class-switching $(36,40)$ was found to be regulated by IL-36 in vivo and in vitro, suggesting that $X b p 1$ might be particularly relevant for IL-36R regulation of $\mathrm{B}$ cell and $\mathrm{PC}$ function.

The epidermal barrier defects in $\mathrm{AD}$ skin are thought to contribute to enhanced exposure of environmental allergens that contribute to allergen-specific IgE and the progression to food allergies, allergic rhinitis, and allergic asthma (2). In our experiments, we found that allergen sensitization with $\mathrm{CrA}$ during the $S$. aureus e.c. exposure resulted in enhanced allergic lung inflammation following i.t. CrA challenge that was dependent on IL-36R signaling. This response also required prior skin allergen sensitization because if i.t. allergen challenge (with OVA) was performed without prior skin allergen sensitization, there was no enhanced lung inflammation. These findings provide a mechanism by which the skin barrier defects and enhanced allergen exposure in AD could directly promote serum IgE production and the progression to IgE-mediated allergic diseases.

Finally, from the clinical perspective, anti-IL-36R-blocking antibody treatment in the $S$. aureus e.c. model was effective in inhibiting AD-like skin inflammation and increased serum $\operatorname{IgE}$ levels. Prior results have found that anti-IL-36R mAb treatment decreased the skin inflammation in a similar $S$. aureus e.c. model in mice, imiquimod-induced psoriasis-like skin inflammation in mice, and the skin inflammation in patients with GPP/DITRA (19, $49,50)$. Our data further suggest that anti-IL-36R mAb blockade (or an alternative therapy to block activity of IL-36 cytokines or IL-36R activity) could also inhibit IgE production and potentially IgE-mediated allergic diseases.

Collectively, keratinocyte-derived IL-36 $\alpha$ provides an additional signal beyond Th2 cells and IL-4 that triggered B cell and IgE class-switching, increased serum IgE and allergic lung inflammation. These findings define a previously unrecognized mechanism by which $\mathrm{AD}$ is associated with other allergic diseases and suggests that IL-36R blockade could represent a potential therapeutic target to prevent or treat IgE-mediated allergic diseases.

\section{Methods}

Methods for in situ hybridization (ISH) of human skin biopsy sections, ex-vivo CFU enumeration, and in vitro mouse epidermal keratinocyte cultures are included in the Supplemental Methods.

S. aureus strains. Bioluminescent derivatives of the parental methicillin-resistant $S$. aureus strain, USA300 LAC, a community-acquired methicillin-resistant $S$. aureus (CA-MRSA) clinical isolate originally obtained from an S. aureus infection outbreak in the Los Angeles County (LAC) jail, were used in all experiments. For the e.c. S. aureus exposure model, the bioluminescent derivative LAC4303 was used and was generated as previously described (20). For the $S$. aureus i.d. challenge model, the bioluminescent derivative USA300 LAC::lux was used (23, 24) and was generated as previously described (51). Both strains possess a modified lux operon from Photorhabdus luminescens in the bacterial chromosome so that the lux construct is maintained and all progeny will produce bioluminescent signals without antibiotic selection.

Bacterial preparation. S. aureus bacteria were streaked onto a tryptic soy agar (TSA) plate (tryptic soy broth [TSB] plus 1.5\% bacto agar; BD Biosciences) and grown in a bacterial incubator overnight at $37^{\circ} \mathrm{C}$. Single colonies were picked and cultured in TSB at $37^{\circ} \mathrm{C}$ in a shaking incubator ( $240 \mathrm{rpm}$ ) overnight (18 hours), followed by subculture (1:50 dilution) at $37^{\circ} \mathrm{C}$ for 2 hours to obtain mid-logarithmic phase bacteria. The bacteria were pelleted, washed, and resuspended in sterile PBS at previously optimized inocula of $1 \times 10^{8} \mathrm{CFU} / 100 \mu \mathrm{L}$ (e.c. model, ref. 20 ) and $3 \times 10^{7} \mathrm{CFU} / 100 \mu \mathrm{L}$ (i.d. model, refs. 23, 24). The absorbance $\left(\mathrm{A}_{600}\right)$ was used to estimate the CFU in each inoculum, which was confirmed by overnight culture on TSA plates.

Generation of the new $I L-36 R^{f / f l}$ mouse strain. The IL-36R ${ }^{\mathrm{f} / / \mathrm{l}}$ mouse strain was generated using TurboKnockout Gene Targeting by Cyagen Biosciences. Briefly, homologous loxP arms flanking exon 3 (in introns 2 and 3) of the mouse IL-36R gene (IL1rl2) were included in a targeting vector using BAC clones RP24-274L12 and RP23-235L22 from the C57BL/6 library as a template. In the targeting vector, the neomycin cassette was flanked by self-deletion anchor sites and the diphtheria toxin fragment A (DTA) gene was included in the $5^{\prime}$ region for negative selection of C57BL/6 embryonic stem (ES) cells. The linearized vector was transfected into C57BL/6 ES cells according to Cyagen's standard electroporation procedures. The transfected ES cells were subject to G418 (Geneticin) selection $(200 \mu \mathrm{g} / \mathrm{mL}) 24$ hours after electroporation and screened by long-range PCR, and verified for cor- 
rect gene targeting by Southern blot and Sanger sequencing. Targeted ES cell clones were injected into C57BL/6 albino embryos, which were then reimplanted into CD-1 pseudo-pregnant females. Founder animals were identified by their coat color, and their germline transmission was confirmed by breeding with C57BL/ 6 females and subsequent genotyping of the offspring. The IL-36 $\mathrm{R}^{\mathrm{fl} / \mathrm{fl}}$ mice on the C57BL/6 background that were homologous for the reporter allele (which was verified again by sequencing) were viable, overtly normal, and had normal IL-36R-signaling function. Following cre-mediated recombination, IL-36R-signaling activity was abolished due to a frameshift of the IL1rl 2 gene. For genotyping cre mice crossed to the IL-36R $\mathrm{R}^{\mathrm{fl} / \mathrm{fl}}$ mice, quantitative real-time PCR (Q-PCR) primers and probes were generated (TransnetYX) to detect the WT IL1rl2 gene (forward: AGATTGCTGCCATTTCCGAAGAT; reverse: TGTTTGAATGATCTAGTTGGGTCTGG; probe: ATCAGCCAGTTTTCC), the floxed allele of the IL1rl2 gene (forward: AGATTGCTGCCATTTCCGAAGAT; reverse: ACTTAAGTGCCGCGGTTGT; probe: TTAAACTTCCAAAACCATTAGCC), and the frameshift mutation of the IL1rl2 gene after cremediated recombination (forward: GTCCAAAACTACCAGAGAGTACAAGTT; reverse: GCTGATTCCTTATCAGATGTGTTTGAATG; probe: ACCAACTGGCTGATCCAG).

Mice. Sex- and age-matched 6- to 10-week-old mice on a C57BL/6 or $\mathrm{BALB} / \mathrm{c}$ background were used for all experiments. C57BL/6 WT mice, BALB/c WT mice, $\beta \delta^{-/-}$mice (lacking $\alpha \beta$ and $\gamma \delta$ T cells (B6.129P2$\left.\mathrm{Tcrb}^{\text {tm1Mom }} \mathrm{Tcrd}^{\mathrm{tm} 1 \mathrm{Mom}} / \mathrm{J}\right)$, and CD4-cre (B6.Cg-Tg(Cd4-cre)1Cwi/BfluJ) mice were obtained from Jackson Laboratories. IL-36R $\mathrm{R}^{-/-}$mice were obtained through a material transfer agreement (MTA) from Amgen. IL-33-- mice on a BALB/c background were obtained through an MTA with AstraZeneca and were generated as previously described (20). $\mathrm{IL}-1 \beta^{--}$mice were obtained through an MTA from Yoichiro Iwakura (University of Tokyo, Japan). CD4-cre mice were crossed to IL-36R $\mathrm{R}^{\mathrm{f} / \mathrm{fl}}$ mice to generate $\mathrm{CD} 4$-cre $\times$ IL-36R $\mathrm{R}^{\mathrm{f} / \mathrm{l}}$ mice, which lack IL-36R signaling in CD4-expressing cells, including both $\mathrm{CD}^{+}$and $\mathrm{CD}^{+} \mathrm{T}$ cells (due to dual expression of CD4 in both T cell types during thymic development).

Mouse model of S. aureus e.c. exposure and disease score measurement. A mouse model of $S$. aureus e.c. exposure was used, which was modified from previous reports (19-21). Briefly, the dorsal skin of anesthetized mice ( $2 \%$ isoflurane) was shaved and depilated (Nair cream) 1 day prior to inoculation. A quantity of $1 \times 10^{8} \mathrm{CFU}$ (USA300 LAC4303) in $100 \mu \mathrm{L}$ PBS was pipetted onto a sterile gauze pad $(1 \times 1 \mathrm{~cm})$, which was applied with on overlying bio-occlusive dressing (Tegaderm; $3 \mathrm{M})$ to the shaved and depilated back skin of mice, and secured with 2 layers of adhesive bandages (BAND-AID, Johnson \& Johnson) for 7 days. The severity of skin inflammation was determined by a blinded observer from digital photographs and was quantified using a disease score: the sum of 3 individual grades for erythema (graded: $0,1,2,3$ ), edema (graded: $0,1,2,3$ ), and scaling/erosion (scaling graded: $0,1,2$, 3 and erosion graded: $4,5,6,7$ ), for a total range of 0-13.

Mouse model of S. aureus i.d. infection and measurement of total lesion size. A mouse model of $S$. aureus i.d. infection was performed as previously described $(23,24)$. Briefly, the dorsal skin of anesthetized mice ( $2 \%$ isoflurane) was shaved and $3 \times 10^{7} \mathrm{CFU}$ (USA300 LAC::lux) in 100 $\mu \mathrm{L}$ PBS was injected intradermally. Total lesion size $\left(\mathrm{cm}^{2}\right)$ was measured from digital photographs using the image analysis software Image J (http://imagej.nih.gov/ij/; NIH) with a millimeter ruler as a reference.

Mouse model of skin allergen exposure and subsequent allergic lung inflammation. A CrA allergen-induced lung inflammation model in mice was modified to first include CrA skin sensitization with or without $S$. aureus e.c. exposure from previously described methods (43, 44). Briefly, CrA (100 $\mu \mathrm{g} / 100 \mu \mathrm{L}$; Greer Laboratories) or PBS $(100 \mu \mathrm{L})$ was applied to the back skin with or without the $S$. aureus e.c. model for 7 days, as described above. On days 10,11, and 12, all mice underwent an i.t. inhalation challenge of $\mathrm{CrA}(20 \mu \mathrm{g} / 50 \mu \mathrm{L}$ PBS $)$. On day 10 , serum was collected via the retro-orbital vein to measure serum IgE levels. Body weights were measured on days 0 and 13 and the percentage of change in body weights was reported. On day 13 , all mice were euthanized and bronchoalveolar lavage (BAL) fluid was harvested by means of 2 consecutive flushes of the lung tissues with $800 \mu \mathrm{L}$ PBS. Lung tissues were then inflated and fixed with $10 \%$ formalin, dissected, and embedded in paraffin for histologic analysis (see below). To enumerate the number of neutrophils, eosinophils, and basophils in the BAL fluid, the BAL fluid was centrifuged $(1500 \mathrm{~g})$ at $4^{\circ} \mathrm{C}$ for $5 \mathrm{~min}$ utes and washed with PBS containing 1\% FBS. Red blood cells in the pellet were lysed with ACK (ammonium-chloride-potassium) lysing buffer. The cells were labeled with mAb for neutrophils, eosinophils, and basophils and flow cytometry was performed (see below). In some experiments, instead of i.t. CrA challenge, on days 10, 11, and 13, mice received i.t. OVA challenge $(20 \mu \mathrm{g} / 50 \mu \mathrm{L} \mathrm{PBS})$ without prior OVA allergen sensitization to the skin.

Histologic analysis of mouse skin and lung. Skin punch biopsy specimens $(10 \mathrm{~mm})$ were collected, fixed in formalin (10\%), and embedded in paraffin. Lungs were inflated and fixed in formalin (10\%) and embedded in paraffin. Sections $(4 \mu \mathrm{m})$ were mounted onto glass slides and stained with H\&E by the Johns Hopkins Reference Histology Laboratory. To measure epidermal thickness, at least 10 epidermal thickness measurements per mouse were averaged from images taken at $\times 200$ magnification (Leica, DFC495) using ImageJ software (NIH). Lung inflammation reported as the percentage of the density of H\&E staining was measured by thresholding cellular region versus open space within the lung tissue in at least 15 regions of lung tissue per mouse averaged from images taken at $\times 200$ magnification (Leica, DFC495) using Image J software.

Mouse serum immunoglobulin levels. Mouse blood was collected on the indicated days from the retro-orbital vein of anesthetized mice (2\% isoflurane) or cardiac puncture of euthanized mice. Sera were then collected after centrifuging the clotted blood (1500g) for 10 minutes at $4^{\circ} \mathrm{C}$. Serum samples were stored at $-80^{\circ} \mathrm{C}$ prior to using in experiments. Mouse serum IgG1 levels were quantified using a mouse IgG1 ELISA kit, according to the manufacturer's instructions (Sigma-Aldrich). Mouse serum IgE and IgG2a levels were quantified using their respective Ready SET-Go! ELISA kits, according to the manufacturer's instructions (Invitrogen).

In vivo bioluminescence imaging (BLI). For the $S$. aureus i.d. mod$\mathrm{el}$, whole animal in vivo bioluminescence imaging was performed on anesthetized mice (2\% isoflurane) using a Lumina III IVIS (PerkinElmer) and total flux (photons/s) was measured within a $1 \times 10^{3}$ pixel square region of interest using Living Image software (PerkinElmer; limit of detection: $2 \times 10^{4}$ photons/s). The bioluminescent signals detected from $S$. aureus (USA300 LAC::lux) infected mice closely approximates ex vivo CFU enumeration $\left(R^{2}=0.9996\right)(52)$.

Immunofluorescence microscopy. Immunofluorescence microscopy for mouse IL-36 $\alpha$ was performed on deparaffinized histologic sections after heat-mediated antigen-retrieval in Trilogy buffer (Cell Marque). Sections were blocked for 1 hour at room temperature in PBS with $10 \%$ 
goat serum (blocking buffer) then incubated at $4^{\circ} \mathrm{C}$ overnight with goat anti-mouse IL-36 $\alpha$ (R\&D Systems) $(2 \mu \mathrm{g} / \mathrm{mL}$ blocking buffer). The next day, sections were incubated for 1 hour at room temperature with FITC Streptavidin $(0.5 \mu \mathrm{g} / \mathrm{mL}$ blocking buffer; BioLegend) and subsequently mounted in Vectashield with DAPI stain (Vector Labs). Fluorescence images were taken at $\times 400$ magnification with AlexaFluor-488 and DAPI signals overlaid using a fluorescence microscope (DFC365FX, Leica Microsystems).

RNA isolation and Q-PCR. Skin punch biopsy $(10 \mathrm{~mm}$ ), dLNs (axillary and brachial), in vitro-cultured mouse epidermal keratinocytes, and in vitro-cultured B cells were snap-frozen in liquid nitrogen and homogenized (Pro200 Series homogenizer; Pro Scientific) in TRIzol reagent. RNA isolation was performed using the Direct-zol RNAMini Prep kit according to the manufacturer's instructions (Zymo Research). cDNA was synthesized using a High Capacity RNA-tocDNA Kit according to the manufacturer's instructions (Applied Biosystems). Commercially available primers and probes for mouse $I L-36 \alpha$,

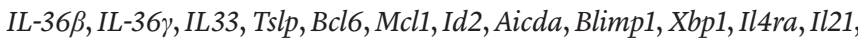
Cd4Olg, Il9, Stat3, Zfp341, and the Taqman Gene Expression Master Mix were used (Applied Biosystems), and Q-PCR was performed using a CFX96 Real-Time PCR System (Bio-Rad). The relative quantities of mRNA per sample were determined using the $\Delta \Delta \mathrm{C}(\mathrm{T})$ formula.

Flow cytometry. A single-cell suspension of dLNs, spleen, or Peyer's patches was obtained by manually pushing the tissues through a cell separation filter $(40 \mu \mathrm{m})$ and resuspending in RPMI containing FBS (10\%), penicillin (100 U/mL), and streptomycin $(100 \mu \mathrm{g} /$ $\mathrm{mL})$. For immunophenotyping of B cells $\left(\mathrm{CD} 19^{+} \mathrm{B} 220^{+}\right)$, GC B cells $\left(\mathrm{CD}^{+} 5^{+} \mathrm{GL}^{+}\right)$, and PCs $\left(\mathrm{CD} 138^{+} \mathrm{FSC}^{\text {hi }}\right)$, cells were stained for viability (Viobility 405/520 viability kit, Miltenyi Biotec) followed by labeling for surface markers with mAbs against B220 (clone RA3-6B2, Miltenyi Biotec), CD19 (clone REA104, Miltenyi Biotec), GL7 (clone GL7, BioLegend), CD95 (clone SA367H8, BioLegend), and CD138 (cloneREA104, Miltenyi Biotec). For flow cytometry analysis of IgE, modified intracellular staining method was used as previously described (53). Briefly, cell surface IgE was first blocked by incubating the cells with a high concentration of mAbs against IgE (clone RME-1, BioLegend), and then stained for viability (Viobility 405/520 viability kit, Miltenyi Biotec). The surface labeled cells were washed and fixed using FACSFix (BD Biosciences) for $30 \mathrm{~min}$ at $4^{\circ} \mathrm{C}$, and permeabilized by incubating for 10 minutes in Perm/Wash (BD Biosciences). Cells were then incubated with mAbs against IgE (clone RME-1, BioLegend) using a low concentration and IgG1 (clone RMG1-1, BioLegend) for 30 minutes at $4^{\circ} \mathrm{C}$ before being washed and resuspended. For immunophenotyping of $\mathrm{Tfh}$ cells $\left(\mathrm{CXCR} 5^{+} \mathrm{PD}-1^{+}\right)$, cells were stained for viability (propidium iodide, Miltenyi Biotec), followed by labeling for surface markers with mAbs against CD45 (30-F11, BD Biosciences), CD4 (clone GK1.5, Miltenyi Biotec), PD-1 (clone HA2-7B1, Miltenyi Biotec), and CXCR5 (clone REA215, Miltenyi Biotec). For detecting IL-4-producing T cells, $1 \times 10^{6}$ cells per well were plated in 96-well cell culture plates with or without a cell activation cocktail of PMA (20 $\mathrm{ng} / \mathrm{mL}$ ) and ionomycin $(1 \mu \mathrm{g} / \mathrm{mL})$ (eBioscience) and incubated at $4^{\circ} \mathrm{C}$ overnight, adding GolgiStop (BD Biosciences) for the last 2 hours. Cells were washed before staining for viability (Viability Fixable Dye, Miltenyi Biotec) and labeled using mAbs against TCR $\gamma \delta$ (clone GL3) and CD4 (clone GK1.5, Miltenyi Biotec) for 30 minutes at $4^{\circ} \mathrm{C}$. Then, the cells were labeled intracellularly with an anti-IL-4 mAb (clone 11B11, BioLegend). For immunophenotyping of neutrophils $\left(\mathrm{Ly}_{6} \mathrm{G}^{+}\right.$,
$\left.\mathrm{CD}_{\left.11 \mathrm{~b}^{+}\right) \text {, eosinophils }(\text { SiglecF }} \mathrm{CD}^{+} \mathrm{CD}^{+}\right)$, and basophils $\left(\mathrm{CD} 49 \mathrm{~b}^{+}\right.$, $\left.\mathrm{CD}_{11 \mathrm{~b}^{+}}\right)$in BAL fluid, fluids were centrifuged $(1500 \mathrm{~g})$ at $4^{\circ} \mathrm{C}$ for $5 \mathrm{~min}-$ utes and washed with PBS containing 1\% FBS. Cells were stained for viability (Viobility 405/520 viability kit, Miltenyi Biotec), followed by labeling for surface markers with mAbs against CD45 (clone 30-F11, BD) CD11b (clone M1/70, BioLegend), and Ly6G (clone 1A8, BioLegend). Cell acquisition was performed on a MACSQuant flow cytometer (Miltenyi Biotec) and data analyzed using FlowJo software (Treestar).

Lymphocyte isolation and adoptive transfer to mice. Lymphocyte isolation from dLNs and adoptive transfer to mice was performed as previously described (23). dLNs were harvested from naive IL-36R $\mathrm{R}^{-/-}$ and WT mice and a single-cell suspension of dLN cells was obtained by manually pushing the dLNs through a cell separation filter (40 $\mu \mathrm{m})$. Cells were counted and resuspended in MACS Separation Buffer (degassed PBS containing BSA [0.5\%] and EDTA [2 mM]) (Miltenyi Biotec). $\mathrm{CD}^{+} \mathrm{T}$ cells were obtained using the Mouse $\mathrm{CD} 4^{+} \mathrm{T}$ cell Isolation Kit, according to the manufacturer's instructions (Miltenyi Biotec). Briefly, non-T cells were depleted using a biotin-antibody cocktail of CD8a, CD11b, CD11c, CD19, CD45R (B220), CD49b (DX5), CD105, anti-MHC-class II, Ter-119, and TCR $\gamma \delta$, labeling with anti-biotin microbeads. The remaining T cells were collected via flow through on the MACS magnetic bead column. $\gamma \delta$ T cells were obtained using the Mouse $\gamma \delta$ T cell Isolation Kit, according to the manufacturer's instructions (Miltenyi Biotec). Briefly, CD11b and CD45R (B220) MicroBeads were used to deplete CD11b+ and CD45R (B220) ${ }^{+}$cells followed by anti-TCR $\gamma \delta$-biotin and anti-biotin microBeads for positive selection of TCR $\gamma \delta^{+} \mathrm{T}$ cells. A quantity of $3 \times 10^{6} \mathrm{CD} 4^{+} \mathrm{T}$ cells or $5 \times 10^{4}$ $\mathrm{TCR} \gamma \delta^{+} \mathrm{T}$ cells were transferred to mice i.v. (via the retro-orbital vein) at 1 week prior to performing the $S$. aureus e.c. model in $\beta \delta^{-/-}$mice.

In vivo antibody administration. To deplete $\mathrm{CD} 4^{+} \mathrm{T}$ cells, mice were treated with anti-CD4 mAb (clone GK1.5, BioXCell) $100 \mu \mathrm{g} / 200$ $\mu \mathrm{L}$ i.p. on day -1 of the $S$. aureus e.c. model, as previously described (23). For IL-4 neutralization, mice were treated with anti-IL-4 mAb (clone 11B11, BioXCell) $100 \mu \mathrm{g} / 200 \mu \mathrm{L}$ sterile PBS i.p. on days $-1,0$, 1 , and sequentially every other day through day 7 of the $S$. aureus e.c. model, according to the manufacturer's instructions (BioXCell). For IL-36R blocking, mice were treated with anti-IL-36R mAb (Boehringer Ingelheim) $250 \mu \mathrm{g} / 200 \mu \mathrm{L}$ sterile PBS i.p. beginning on days $-1,0$, 1 , and sequentially every other day through day 7 of the $S$. aureus e.c. model, as previously described (54). For TSLP neutralization, mice were treated with anti-TSLP antibody (clone 28F12, Thermo Fisher Scientific) $200 \mu \mathrm{g} / 200 \mu \mathrm{L}$ sterile PBS i.p. on days 0,3 , and 7 of the S. aureus e.c. model, as previously described (28). Control mice were treated with a corresponding isotype control antibody at equivalent doses and time points.

In vitro B cell cultures, ELISpot, and proliferation assays. In vitro B cell antibody-producing conditions were modified from previously reported methods $(55,56)$. Briefly, spleens, axillary lymph nodes, and inguinal lymph nodes from naive WT mice were obtained. After manually pushing them through a cell separation filter $(40 \mu \mathrm{m})$, red blood cells were lysed with ACK lysis buffer and B cells were isolated using a murine Pan-B cell Isolation Kit (Miltenyi Biotec). B cells were then plated in 24-well plates (Costar) in a volume of $1 \mathrm{~mL}$ complete RPMI 1640 (containing extra L-glutamine and 2-mercaptoethanol) with anti-CD40 mAb $(1 \mu \mathrm{g} / \mathrm{mL}$, Thermo Fisher Scientific), anti-IgM $\mathrm{mAb}(10 \mu \mathrm{g} / \mathrm{mL}$, Thermo Fisher Scientific), recombinant IL-4 (rIL-4) (20 ng/mL, Thermo Fisher Scientific), heat-killed S. aureus (HKSA) 
$\left(1 \times 10^{6} \mathrm{CFU}\right)$ with or without rIL-36 $\alpha(20 \mathrm{ng} / \mathrm{mL}$; cleaved, aa 8-160, R\&D Systems) and cultured for 5 days. Mouse IgE ELISpot assays were performed on cultured B cells using a Mouse IgE ELISpot Kit, according to manufacturer's standard protocols (Mabtech). The number of IgE ELISpots were enumerated using the AID iSpot Spectrum reader (Autoimmun Diagnostika $\mathrm{GmbH}$ ). In some experiments, the cultured B cells were labeled with $5 \mu \mathrm{M}$ CFSE (Invitrogen) prior to adding the conditions above and culturing for 3 days. CFSE dilution was measured by flow cytometry using a MACSQuant flow cytometer (Miltenyi Biotec) and data analyzed using FlowJo software (Treestar).

Human serum immunoglobulin and protein levels. Human serum IgE levels from healthy humans and patients with $\mathrm{AD}$ were measured by the NIH Clinical Center Diagnostic Lab by nephelometry, according to guidelines for clinical specimens. Human serum IL-36 $\alpha$ and IL-36 lev-

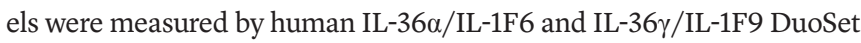
ELISA kits, according to the manufacturer's instructions (R\&D Systems).

Statistics. Data from single comparisons were analyzed by a 2-tailed Student's $t$ test or 2-way ANOVA, and multiple comparisons were analyzed by a 1-way ANOVA with the Tukey correction, as indicated in the figure legends. All statistical analyses were performed using Prism software version 8 (GraphPad). Data are presented as mean \pm SEM and values of $P$ less than 0.05 were considered statistically significant.

Study approval. All mouse strains were bred and maintained under the same specific pathogen-free conditions at a Johns Hopkins University animal facility accredited by the American Association for the Accreditation of Laboratory Animal Care (AAALAC), and housed according to procedures described in the Guide for the Care and Use of Laboratory Animals (National Academies Press, 2011). Human subject blood and skin samples were gathered from the Johns Hopkins Cutaneous Translational Research Program (CTReP) unit approved by the Johns Hopkins University IRB (including healthy human subjects [ $n=$ 3] and patients with $\mathrm{AD}[n=4])$. Human subject blood samples were also gathered at the NIH Clinical Center approved by the National Institute of Allergy and Infectious Diseases IRB (including healthy human subjects [ $n=14]$, patients with $\mathrm{AD}[n=29]$, and a patient with
DITRA $[n=1])$. For the in situ hybridization experiments, healthy human skin resections obtained from healthy humans undergoing cosmetic skin procedures $(n=4)$ and skin punch biopsy specimens from patients with $\mathrm{AD}(n=5)$ were prospectively acquired from Discovery Life Sciences. All human subjects gave written informed consent.

\section{Author contributions}

GJP, HL, MPA, DAD, CY, JCO, YW, AR, MM, GD, RVO, EZ, RJM, CAD, QL, SJN, KN, LM, EMW, DCD, YZ, GS, AIM, and AAM performed and designed experiments and analyzed data. GC, ACK, DVM, JSF, MR, and ELR contributed reagents. GC, RGM, and JDM provided clinical samples. GJP, HL, EM, JDM, LAG, PG, MR, ELR, NKA, and LSM conceived the study, designed experiments, interpreted data and wrote the manuscript.

\section{Acknowledgments}

This study was funded in part by NIH grants R01AR073665 (to LSM and NKA) and R01AR069502 (to LSM and NKA) and an Investigator Initiated Study from Boehringer Ingelheim (to LSM and NKA). The content is solely the responsibility of the authors and does not necessarily represent the official views of the NIH. We thank Tammy Kielian (University of Nebraska, Lincoln, Nebraska, USA) for providing the bioluminescent USA300 LAC::lux S. aureus strain, AstraZeneca (Cambridge, United Kingdom) for providing the IL-33 $3^{-/-}$mice, and Yoichiro Iwakura (University of Tokyo, Tokyo, Japan) for providing the IL-1 $\beta^{-/-}$mice.

Address correspondence to: Nathan K. Archer or Lloyd S. Miller, Johns Hopkins Department of Dermatology, Cancer Research Building II, Suite 209, 1550 Orleans Street, Baltimore, Maryland 21231, USA. Phone: 410.955.8662; Email: narcher2@jhmi.edu (NKA); lloydmiller@jhmi.edu (LSM).

LSM's present address is: Immunology, Janssen Research and Development, Pennsylvania, USA.
1. Murrison LB, et al. Environmental exposures and mechanisms in allergy and asthma development. J Clin Invest. 2019;129(4):1504-1515.

2. Tham EH, Leung DY. Mechanisms by which atopic dermatitis predisposes to food allergy and the atopic march. Allergy Asthma Immunol Res. 2019;11(1):4-15.

3. Paller AS, et al. The atopic march and atopic multimorbidity: Many trajectories, many pathways. J Allergy Clin Immunol. 2019;143(1):46-55.

4. Davidson WF, et al. Report from the National Institute of Allergy and Infectious Diseases workshop on "Atopic dermatitis and the atopic march: Mechanisms and interventions". JAllergy Clin Immunol. 2019;143(3):894-913.

5. Wu LC, Zarrin AA. The production and regulation of IgE by the immune system. Nat Rev Immunol. 2014;14(4):247-259.

6. Dharmage SC, et al. Atopic dermatitis and the atopic march revisited. Allergy. 2014;69(1):17-27.

7. Czarnowicki T, et al. Atopic dermatitis endotypes and implications for targeted therapeutics.

JAllergy Clin Immunol. 2019;143(1):1-11.

8. Weidinger S, Novak N. Atopic dermatitis. Lancet.
2016;387(10023):1109-1122.

9. Kim J, et al. Interactions between atopic dermatitis and staphylococcus aureus infection: clinical implications. Allergy Asthma Immunol Res. 2019;11(5):593-603.

10. Geoghegan JA, et al. Staphylococcus aureus and atopic dermatitis: a complex and evolving relationship. Trends Microbiol. 2018;26(6):484-497.

11. Williams MR, et al. Staphylococcus aureus: master manipulator of the skin. Cell Host Microbe. 2017;22(5):579-581.

12. Imai Y, et al. Skin-specific expression of IL-33 activates group 2 innate lymphoid cells and elicits atopic dermatitis-like inflammation in mice. Proc Natl Acad Sci U S A. 2013;110(34):13921-13926.

13. Leyva-Castillo JM, et al. Mechanical skin injury promotes food anaphylaxis by driving intestinal mast cell expansion. Immunity. 2019;50(5):1262-1275.

14. Pattarini L, et al. TSLP-activated dendritic cells induce human $\mathrm{T}$ follicular helper cell differentiation through OX40-ligand. J Exp Med. 2017;214(5):1529-1546.

15. Noti M, et al. Exposure to food allergens through inflamed skin promotes intestinal food allergy through the thymic stromal lymphopoietin-basophil axis. J Allergy Clin Immunol. 2014;133(5):1390-1399.

16. Zhang Z, et al. Thymic stromal lymphopoietin overproduced by keratinocytes in mouse skin aggravates experimental asthma. Proc Natl Acad Sci U S A. 2009;106(5):1536-1541.

17. Ray RJ, et al. Characterization of thymic stromal-derived lymphopoietin (TSLP) in murine B cell development in vitro. Eur J Immunol. 1996;26(1):10-16.

18. Stier MT, et al. IL-33 is a cell-intrinsic regulator of fitness during early B cell development. J Immunol. 2019;203(6):1457-1467.

19. Nakagawa S, et al. Staphylococcus aureus virulent psm $\alpha$ peptides induce keratinocyte alarmin release to orchestrate IL-17-dependent skin inflammation. Cell Host Microbe. 2017;22(5):667-677.

20. Liu H, et al. Staphylococcus aureus epicutaneous exposure drives skin inflammation via IL-36mediated T cell responses. Cell Host Microbe. 2017;22(5):653-666. 
21. Nakamura Y, et al. Staphylococcus $\delta$-toxin induces allergic skin disease by activating mast cells. Nature. 2013;503(7476):397-401.

22. Xu Z, et al. Immunoglobulin class-switch DNA recombination: induction, targeting and beyond. Nat Rev Immunol. 2012;12(7):517-531.

23. Dillen CA, et al. Clonally expanded $\gamma \delta \mathrm{T}$ cells protect against Staphylococcus aureus skin reinfection. J Clin Invest. 2018;128(3):1026-1042.

24. Marchitto MC, et al. Clonal $\mathrm{V} \gamma 6^{+} \mathrm{V} \delta 4^{+} \mathrm{T}$ cells promote IL-17-mediated immunity against Staphylococcus aureus skin infection. Proc Natl Acad Sci US A. 2019;116(22):10917-10926.

25. Bassoy EY, et al. Regulation and function of interleukin-36 cytokines. Immunol Rev. 2018;281(1):169-178.

26. Al Kindi A, et al. Staphylococcus aureus second immunoglobulin-binding protein drives atopic dermatitis via IL-33 [published online October 1, 2020]. J Allergy Clin Immunol. https://doi. org/10.1016/j.jaci.2020.09.023.

27. Takai T, et al. TSLP expression induced via Tolllike receptor pathways in human keratinocytes. Methods Enzymol. 2014;535:371-387.

28. Mosconi I, et al. Intestinal bacteria induce TSLP to promote mutualistic T-cell responses. Mucosal Immunol. 2013;6(6):1157-1167.

29. Walker JA, McKenzie ANJ. $\mathrm{T}_{\mathrm{H}} 2$ cell development and function. Nat Rev Immunol. 2018;18(2):121-133.

30. Martel BC, et al. Distinct molecular signatures of mild extrinsic and intrinsic atopic dermatitis. Exp Dermatol. 2016;25(6):453-459.

31. Suárez-Fariñas $\mathrm{M}$, et al. RNA sequencing atopic dermatitis transcriptome profiling provides insights into novel disease mechanisms with potential therapeutic implications. J Allergy Clin Immunol. 2015;135(5):1218-1227.

32. Tsoi LC, et al. Progression of acute-to-chronic atopic dermatitis is associated with quantitative rather than qualitative changes in cytokine responses. JAllergy Clin Immunol. 2019;145(5):1406-1415.
33. Pan J, et al. Juvenile generalized pustular psoriasis with IL36RN mutation treated with short-term infliximab. Dermatol Ther. 2016;29(3):164-167.

34. Zea-Vera AF, et al. Interleukin-36 receptor antagonist deficiency (DITRA) with a novel IL36RN homozygous mutation c.200G > T (P.Cys67Phe) in a young Colombian woman. JClin Immunol. 2019;39(3):261-263.

35. Akdis M, Akdis CA. IgE class switching and cellular memory. Nat Immunol. 2012;13(4):312-314.

36. Todd DJ, et al. XBP1 governs late events in plasma cell differentiation and is not required for antigen-specific memory B cell development. JExp Med. 2009;206(10):2151-2159.

37. Tong P, Wesemann DR. Molecular mechanisms of IgE class switch recombination. Curr Top Microbiol Immunol. 2015;388:21-37.

38. Wu YL, et al. Intrinsic transcriptional heterogeneity in B cells controls early class switching to IgE. JExp Med. 2017;214(1):183-196.

39. Yang $Z$, et al. IL-21 is a broad negative regulator of IgE class switch recombination in mouse and human B cells. JExp Med. 2020;217(5):e20190472.

40. Zhang Y, et al. Global gene regulation during activation of immunoglobulin class switching in human B cells. Sci Rep. 2016;6:37988.

41. Fawaz LM, et al. Expression of IL-9 receptor alpha chain on human germinal center $B$ cells modulates IgE secretion. JAllergy Clin Immunol. 2007;120(5):1208-1215.

42. Harusato A, et al. IL-36 $\gamma$ signaling controls the induced regulatory $\mathrm{T}$ cell-Th 9 cell balance via $\mathrm{NF} \kappa \mathrm{B}$ activation and STAT transcription factors. Mucosal Immunol. 2017;10(6):1455-1467.

43. Do DC, et al. Cockroach allergen exposure and risk of asthma. Allergy. 2016;71(4):463-474.

44. Gao P, et al. Functional effects of TGF- $\beta 1$ on mesenchymal stem cell mobilization in cockroach allergen-induced asthma. JImmunol. 2014;192(10):4560-4570.

45. Cho JS, et al. Noninvasive in vivo imaging to evaluate immune responses and antimicrobial therapy against Staphylococcus aureus and USA300 MRSA skin infections. J Invest Dermatol. 2011;131(4):907-915.

46. Johnston A, et al. IL-1 and IL-36 are dominant cytokines in generalized pustular psoriasis. J Allergy Clin Immunol. 2017;140(1):109-120.

47. Frey S, et al. The novel cytokine interleukin-36 $\alpha$ is expressed in psoriatic and rheumatoid arthritis synovium. Ann Rheum Dis. 2013;72(9):1569-1574.

48. Schmitt $V$, et al. Interleukin-36 receptor mediates the crosstalk between plasma cells and synovial fibroblasts. Eur JImmunol. 2017;47(12):2101-2112.

49. Bachelez H, et al. Inhibition of the interleukin-36 pathway for the treatment of generalized pustular psoriasis. NEngl JMed. 2019;380(10):981-983.

50. Su Z, et al. IL-36 receptor antagonistic antibodies inhibit inflammatory responses in preclinical models of psoriasiform dermatitis. Exp Dermatol. 2019;28(2):113-120.

51. Thurlow LR, et al. Staphylococcus aureus biofilms prevent macrophage phagocytosis and attenuate inflammation in vivo. J Immunol. 2011;186(11):6585-6596.

52. Guo Y, et al. In vivo bioluminescence imaging to evaluate systemic and topical antibiotics against community-acquired methicillin-resistant Staphylococcus aureus-infected skin wounds in mice. Antimicrob Agents Chemother. 2013;57(2):855-863.

53. Yang $Z$, et al. Fluorescent in vivo detection reveals that $\operatorname{IgE}(+) B$ cells are restrained by an intrinsic cell fate predisposition. Immunity. 2012;36(5):857-872.

54. Ganesan R, et al. Generation and functional characterization of anti-human and anti-mouse IL-36R antagonist monoclonal antibodies. MAbs. 2017;9(7):1143-1154.

55. Ettinger R, et al. IL-21 induces differentiation of human naive and memory $B$ cells into antibody-secreting plasma cells. J Immunol. 2005;175(12):7867-7879.

56. Janssen E, et al. TRIF signaling is essential for TLR4-driven IgE class switching. J Immunol. 2014;192(6):2651-2658 